\title{
Tempo-spatial chirogenesis. Limonene-induced mirror symmetry breaking of Si-Si bond polymers during aggregation in chiral fluidic media
}

Michiya Fujiki, ${ }^{\dagger *}$ Keisuke Yoshida, ${ }^{\dagger}$ Nozomu Suzuki, ${ }^{\dagger}$ Nor Azura Abdul Rahim, ${ }^{\dagger, \dagger}$ and Jalilah Abd Jalil ${ }^{\dagger} \ddagger$

Corresponding author: e-mail: fujikim@ms.naist.jp

${ }^{\dagger}$ Graduate School of Materials Science, Nara Institute of Science and Technology (NAIST), 8916-5 Takayama, Ikoma, Nara 630-0192, Japan

${ }^{\ddagger}$ School of Materials Engineering, Universiti Malaysia Perlis, 02600, Arau, Perlis, Malaysia

Dedicated to Prof. Yoshihisa Inoue, who discovered entropy driven chirogenesis and is a seeker for the origin of homochirality on the Earth.

\begin{abstract}
Herein, we designed photoluminescent polymer aggregates surrounded by organic media containing $(S)-/(R)$-limonene and $(1 S)-/(1 R)$ - $\alpha$-pinene as an artificial model of an open-flow cell-wall free coacervate in a fluidic medium in the ground and photoexcited states. The aggregates were build-up of stiff circular dichroism (CD)-silent and circularly polarized luminescence (CPL)-silent bis(p-n-butylphenyl)polysilanes, $\boldsymbol{n B u P S}$, and four other diarylpolysilanes. $(S)$ - and $(R)$-limonene induced more efficiently to their chirality to $n \mathbf{B u P S}$ during aggregation, as proven by CD and CPL spectral analysis, compared to $(1 S)$ - and $(1 R)$ - $\alpha$-pinene. The $\boldsymbol{n B u P S}$ aggregates generated in a mixture of limonene, methanol, and chloroform had a dissymmetry factor $\left(g_{\text {abs }}\right)$ as high as +0.04 for $(R)$-limonene and -0.03 for $(S)$-limonene at the first Cotton band and a weak dissymmetry factor $\left(g_{\text {lum }}\right)$ of +0.004 for $(R)$-limonene and -0.003 for $(S)$-limonene. The $g_{\text {abs }}$ factor, however, greatly depended on the volume fraction and chirality of limonene in the tersolvents. These behaviors were ascribed to the tempo-spatial stability and instability of the aggregates suspension in the fluidic media, as revealed by time-course dynamic light scattering measurement.
\end{abstract}

keywords: symmetry breaking; homochirality; far-from-equilibrium; aggregates; circular dichroism; circularly polarized luminescence; 


\section{Introduction}

In astronomy, astrochemistry, astrobiology, and astrophysics, researchers seeking extra-terrestrial life have long assumed that liquid water is inevitably needed for the evolution of life. In 2013, Hubble space telescope (NASA) captured geysers of liquid water from Europa covered with a crust of thick ice, one of the Jupiter family [1]. The most spectacular events in 2014 might be discoveries of several exoplanets located in habitable zones [2-6]. Kepler (NASA) indicated that several habitable exoplanets, named super-earths, exist [2]. Super-earth means that the planet's size is almost comparable to that of our Earth. Kepler found the first super-earth with 1.2-Earth-radius, named Kepler-186f [3]. Tau Ceti e, the nearest super-earth 11.9 light-years away from us, is estimated to have an average surface temperature of $\approx 70{ }^{\circ} \mathrm{C}$ [4]. Moreover, Cassini (a part of Cassini-Huygens missions of NASA) using a quadrupole gravity measurement system discovered water under the surface of Enkelados, one of 67 Saturnian moons [5]. The Jet Propulsion Laboratory at NASA concludes that Titan, the largest Saturnian moon, has extremely salty water involving inorganic salts of sulfur, sodium, and potassium [6]. Rosetta (ESA) invoked the detection of prebiotic constituents involving water, several organic fragments, and $\mathrm{SiO}_{2}$ by a direct landing on comet 67P/Churyumov-Gerasimenko, the Jupiter family [7,8]. In 2015, NASA confirmed that liquid water, though containing $\mathrm{Ca}\left(\mathrm{ClO}_{4}\right)_{2}, \mathrm{Mg}\left(\mathrm{ClO}_{4}\right)_{2}$, and $\mathrm{NaClO}_{4}$ salts, is still flowing on the surface of Mars by the detection of water-related $1.4 \mu \mathrm{m}$ and $1.9 \mu \mathrm{m}$ bands in the near-infrared region [9].

If life existed in the past or is now existing on these exoplanets, solar planets, moons, and comets, a big question with a great curiosity is whether $S$-/R-point chirality (stereogenic centers) and/or $P$ - $/ M$-helicity (stereogenic bonds) are identical to those on our Earth $[10,11]$. Living organisms are always tempo-spatial and metastable as a consequence of far-from-equilibrium open system [12]. Life can survive only under open flows of energy and chemical sources because life eats low-entropy food and solar energy [13].

The origin of homochirality on the Earth has been a mystery in the modern scientific community over 150 years [10,14-24] because life cannot exit without molecular asymmetry [14]. So far, many scientists have proposed several plausible scenarios from the primordial era to answer this big question. Scientists have long argued about the possibility of exo-terrestorial life [10,15-22] as well as the origin of life on Earth. Circularly polarized radiation sources, such as $\gamma$-ray, X-ray, and vacuum UV, may become a trigger for the left-right selection of biomolecular substances [15-22]. In 1969, mankind received an incredible gift of carbonaceous stone, known as the Murchison meteorite, from the interstellar 
Universe [25]. The meteorite was contaminated with terrestrial origin amino acids, of which $L$-enantiomers were in excess over $D$-ones, revealed by a largely enriched ${ }^{15} \mathrm{~N}$ in ${ }^{15} \mathrm{~N} /{ }^{14} \mathrm{~N}$ ratio and marked depletions of ${ }^{13} \mathrm{C}$ in ${ }^{13} \mathrm{C} /{ }^{12} \mathrm{C}$ ratio and $\mathrm{D}$ in $\mathrm{D} / \mathrm{H}$ ratio, relative to the biological origin amino acids on the Earth [26,27]. A recent study demonstrated that a subtle imbalance in $L$-/D-amino acids can catalyze the asymmetric generation of carbohydrates with a high $e e$, which might be responsible for the homochiral world [28]. Moreover, even nearly racemic substances with $10^{-5} \%$ ee can be significantly amplified to nearly $100 \% e e$, as exemplified by the Soai-reaction [29].

The homochirality question, however, is a difficult one because of a complete lack of any fossil record or trace amount of chiral molecular evidence. However, marked depletion of ${ }^{13} \mathrm{C}$ in ${ }^{13} \mathrm{C}-/{ }^{12} \mathrm{C}$-isotopic ratio suggests evidence that methanogenic microbial existed in the Archaean era at least 3.5-3.8 Gyrs ago [30-32]. This conclusion was drawn from the fact that lighter ${ }^{12} \mathrm{C}$-containing substances in living organisms are enriched during their entire lifetime. Moreover, the recent snowball Earth hypothesis with analysis of palaeomagnetism asserts that Prokaryotes only inhabited Precambrian eras for more than 2 Gyrs [33,34], though critical argument has been still made to the radical hypothesis [35].

In 1920s-1930s, a more radical idea was hypothesized by Oparlin in Russia [36] and Haldane in UK [37]. They independently claimed that the coacervate is a prototype of living cells during the chemical evolution of life. The coacervate refers to spherical aggregates surrounded by water - colloidal droplets - made of stable organic matter, followed by spontaneous growing and fermentation. This hypothesis relies on spontaneous association during liquid-liquid phase separation resulting from non-covalent electrostatic, hydrogen bonding, and van der Waals interactions. Coacervate size typically ranges from 1 to $100 \mu \mathrm{m}$ in diameter, almost identical to that of living cells. However, the hypothesis was mostly abandoned because coacervate did not evolve living life. At the time, although colloids made of low-molecular mass organic matter were commonly known among scientists, high-molecular mass molecules exceeding 10,000 proposed by Staudinger were not widely accepted [38]. Nowadays, high molecular mass molecules are commonly known as polymers and macromolecules.

Most hypotheses assumed coacervate to be rigid hard particles surrounded by water without any flows of energy and chemical substances. If coacervates can adopt a more dynamic structure like a swollen gel, this soft matter system should be adaptable to any alterations of external biases, allowing flows of chiral chemical and circularly polarized photon energy sources. This idea led us to design a cell-wall free, semi-artificial coacervate 
model built up from chain-like synthetic polymers surrounded by a mixture of chiral and achiral organic solvents. This open system allows investigation of tempo-spatial molecular chirality transcription of the soft aggregates. Particularly, time-dependent alteration in the size of optically active aggregates becomes measurable because optically active aggregation becomes popular in recent years [39-54].

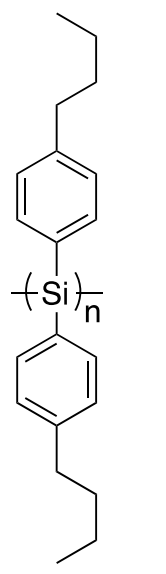

nBuPS

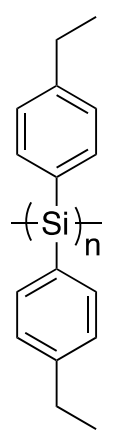<smiles>CC(C)c1ccc([Si](C)(C)c2ccc(C(C)C)cc2)cc1</smiles>

IPrPS

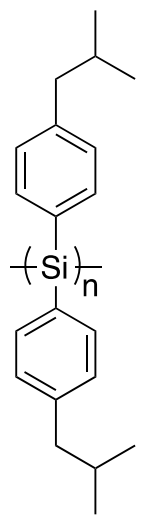

iBuPS

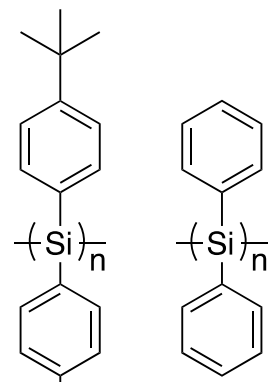

(S)-limonene<smiles>CC1=CC2C3C(C)(C)CC1C3(C)C2(C)C</smiles>

$(1 R)-\alpha$-pinene<smiles>C=C(C)[C@H]1CC=C(C)CC1</smiles>

$(R)$-limonene

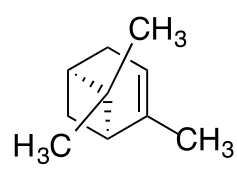

(1S)- $\alpha$-pinene

Fig. 1. Chemical structures of diarylpolysilanes, limonene, and $\square$-pinene used in this work.

Aiming at realizing the soft matter coacervate system enabling open flows of chiral substances in the ground state and/or circularly polarized radiation in the photoexcited state, the following five bis( $p$-alkylphenyl)polysilanes, were chosen: $\boldsymbol{n B u P S}, \mathbf{E t P S}, \boldsymbol{i P r P S}, \boldsymbol{i B u P S}$, and $\boldsymbol{t B u P S}$ (Fig. 1). These Si-Si bond polymers are non-charged chromophores and luminophores made of multiple stereogenic bonds. Although the first synthesis of $\boldsymbol{n B u P S}$ was reported by Miller and Sooriyakumaran three decades ago, $n$ BuPS is shown to adopt a notably stiff backbone with a persistence length $(q)$ of $\approx 10 \mathrm{~nm}$, which is one third relative to the $q$ of double-strand DNA $[55,56]$. The long $q$ value means that $\approx 55$ repeating $\boldsymbol{n B u P S}$ units behave as an ideal rod. The other four EtPS, $\boldsymbol{i}$ PrPS, $\boldsymbol{i}$ BuPS, and $\boldsymbol{t}$ BuPS possibly adopt a similar stiff backbone because they commonly have bulky diaryl groups attached to the backbone. Non-polar $(S)-/(R)$-limonene and $(1 S)-/(1 R)-\alpha$-pinene were chosen as chiral terpenes because limonene and $\alpha$-pinene are efficient stereogenic center transferable solvents for several circular dichroism (CD)-silent and/or circularly polarized light (CPL)-silent $\pi$ - and $\sigma$-conjugated polymers [44,49-54]. CD-active and/or CPL-active aggregation of these polymers occurs with the help of van der Waals force only with these terpenes. Aggregation

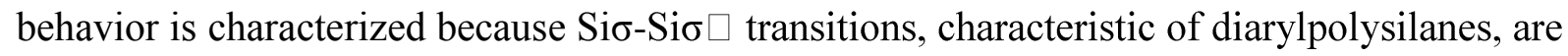


chiroptical probes to detect chiralities in the ground- and photoexcited states of the backbones [56-58]. Compared to $\pi-\pi^{*}$ transitions associated with phonon side bands, very narrow structure-less Si $\sigma-S^{*} \sigma^{*}$ transition spectral bands facilitate to detect subtle imbalance between $P$ - and $M$-motifs and helical pitch, that are detectable by CD and CPL spectroscopies, and dynamic light scattering (DLS) and fluorescence optical microscopic (FOM) methods.

\section{Results and discussion}

\subsection{Inherently CD-silent helical $\boldsymbol{n B u P S}$}

The five diarylpolysilanes are CD-silent and CPL-silent polymeric luminophores. Calculation of a 30-mer model as $\boldsymbol{n B u P S}$ showed the existence of four local minima at $165^{\circ} / 195^{\circ}$ dihedral angle in the ground state, corresponding to $P$ - and $M-15_{7}$ helices, respectively, and $150^{\circ} / 210^{\circ}$ dihedral angles corresponding to $P$ - and $M-7_{3}$ helices, respectively $[57,58]$. The four local minima are due to the para-substitution of alkyl group at the phenyl group. For comparison, from the calculation of the 30-mer model for unsubstituted diphenylpolysilane, PS prefers global minima at $150^{\circ} / 210^{\circ}$ angles, yielding $P$ - and $M-7_{3}$ helices rather than $15_{7}$ helices [57,58] (Fig. 2).

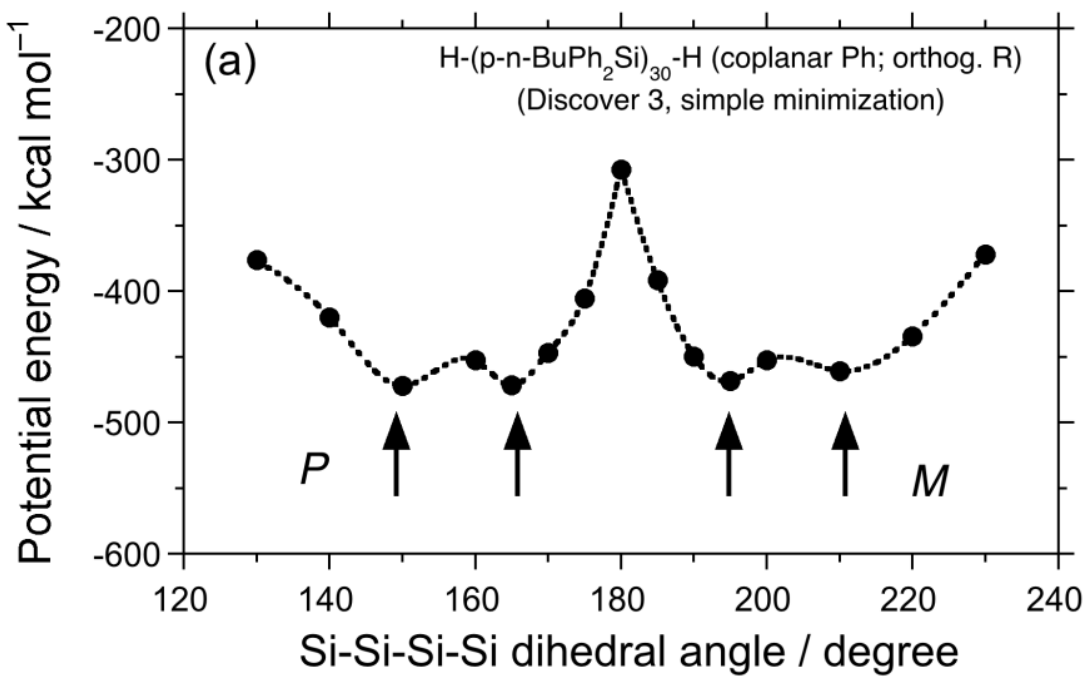




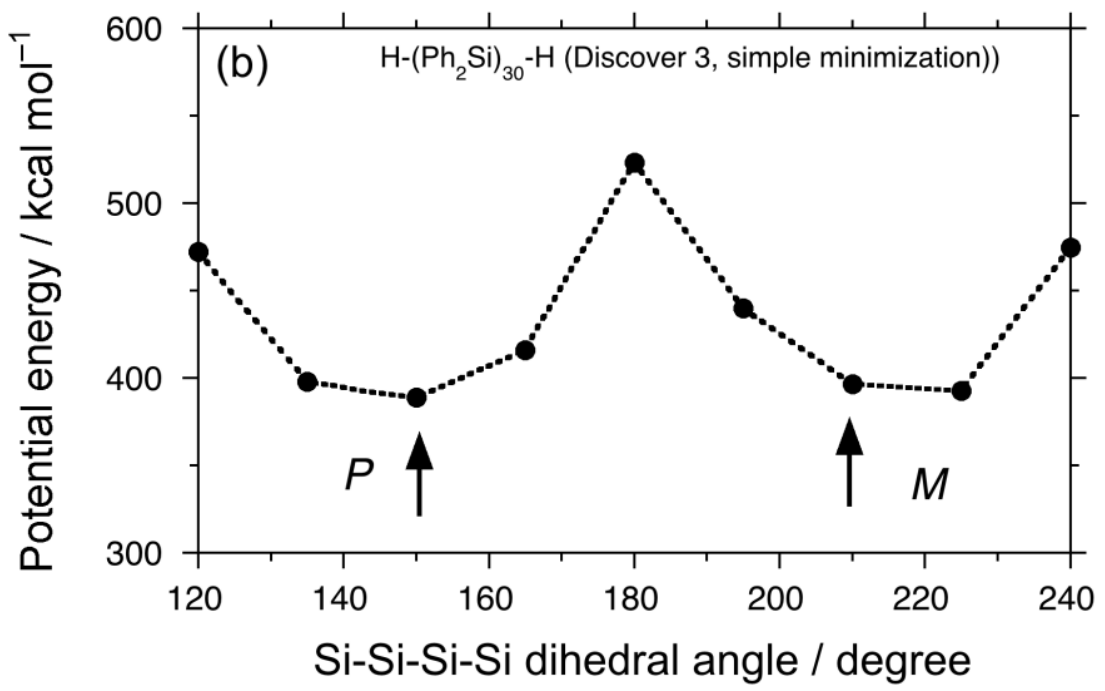

Fig. 2. Energy as a function of dihedral angle in model 30-mers of (a) $\boldsymbol{n B u P S}$ and (b) PS. Data taken from the original and unpublished data ${ }^{58}$, followed by re-generation of potential curves.

The $165^{\circ} / 195^{\circ}$ dihedral sets are responsible for intense excitonic CD-active and CD-silent

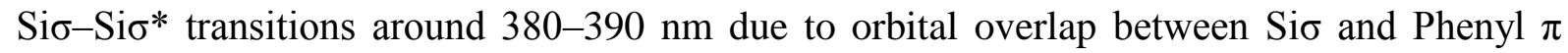

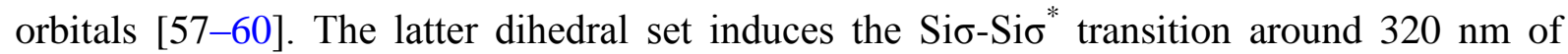
dialkylpolysilanes due to the lack of orbital overlap between Si $\sigma$ and phenyl $\pi$ orbitals $[59,60]$.

$n$ BuPS has a high molecular mass $\left(M_{\mathrm{w}}=85,000, \approx 300\right.$ repeating units $)$, while the other four diarylpolysilanes had a lower molecular mass smaller than 4,000-6,000 (corresponding to $\approx 20$ repeating units). $\boldsymbol{n B u P S}$ and EtPS have non-branched alkyl groups at the para-position of phenyl, while $\boldsymbol{i}$ PrPS, $\boldsymbol{i}$ BuPS, and $\boldsymbol{t}$ BuPS carry para-branched alkyl groups. The substitutes and molecular mass effects in the aggregation are discussed later.

\subsection{Chiroptical properties of $\boldsymbol{n B u P S}$ in homogeneous solutions}

The UV-vis, CD, and PL (excited at $350 \mathrm{~nm}$ ) spectra (wavelength, $\mathrm{nm}$ in unit) of $\boldsymbol{n B u P S}$ in $\mathrm{CHCl}_{3}$ solution are given in Fig. 3a. For comparison, the corresponding UV-vis and PL spectra (photon energy, eV in unit) of $\boldsymbol{n B u P S}$ are given in Appendix Fig. S1a. The Stokes' shift of $\boldsymbol{n B u P S}$ is as small as $0.118 \mathrm{eV}\left(\approx 951 \mathrm{~cm}^{-1}\right)$. The full-width-at-half maxima of UV-vis and PL spectra are $0.146 \mathrm{eV}\left(1180 \mathrm{~cm}^{-1}\right)$ and $0.125 \mathrm{eV}\left(1010 \mathrm{~cm}^{-1}\right)$, respectively. These narrower absorption and emission bands are characteristics of quasi-one-dimensional (Q1D) exciton (photoexcited electron-hole pair) confined in $\mathrm{Si}-\mathrm{Si}$ bond quantum wire. The exciton state in Q1D polysilane wire stably exists with a high binding energy of $\approx 1 \mathrm{eV}$ [61]. The small Stokes' shift indicates a very rigid $\mathrm{Si}-\mathrm{Si}$ bond backbone and a minimal 
reorganization of the backbone in the photoexcited state. These spectral characteristics have been reported previously [58]. However, from the potential energy surface in Fig. 2a, this intense, narrow exciton band at $395 \mathrm{~nm}(3.139 \mathrm{eV})$ is a consequence of $P$ - and $M-15_{7}$ helices that coexist in the same backbone. Energy barrier height between $P$ - and $M$-helices is estimated to be as small as 5-6 kcal per Si repeating unit [57,58]. This rotational barrier height is comparable to the inversion barrier height of molecular ammonium. This uniqueness makes $\boldsymbol{n B u P S}$ alter its preferential helix sense, $P$ or $M$, susceptible to external chiral chemical and photophysical biases. This means that change in global conformation arises from cooperative helix-to-helix transition, not due to rod-to-coil phase transition.

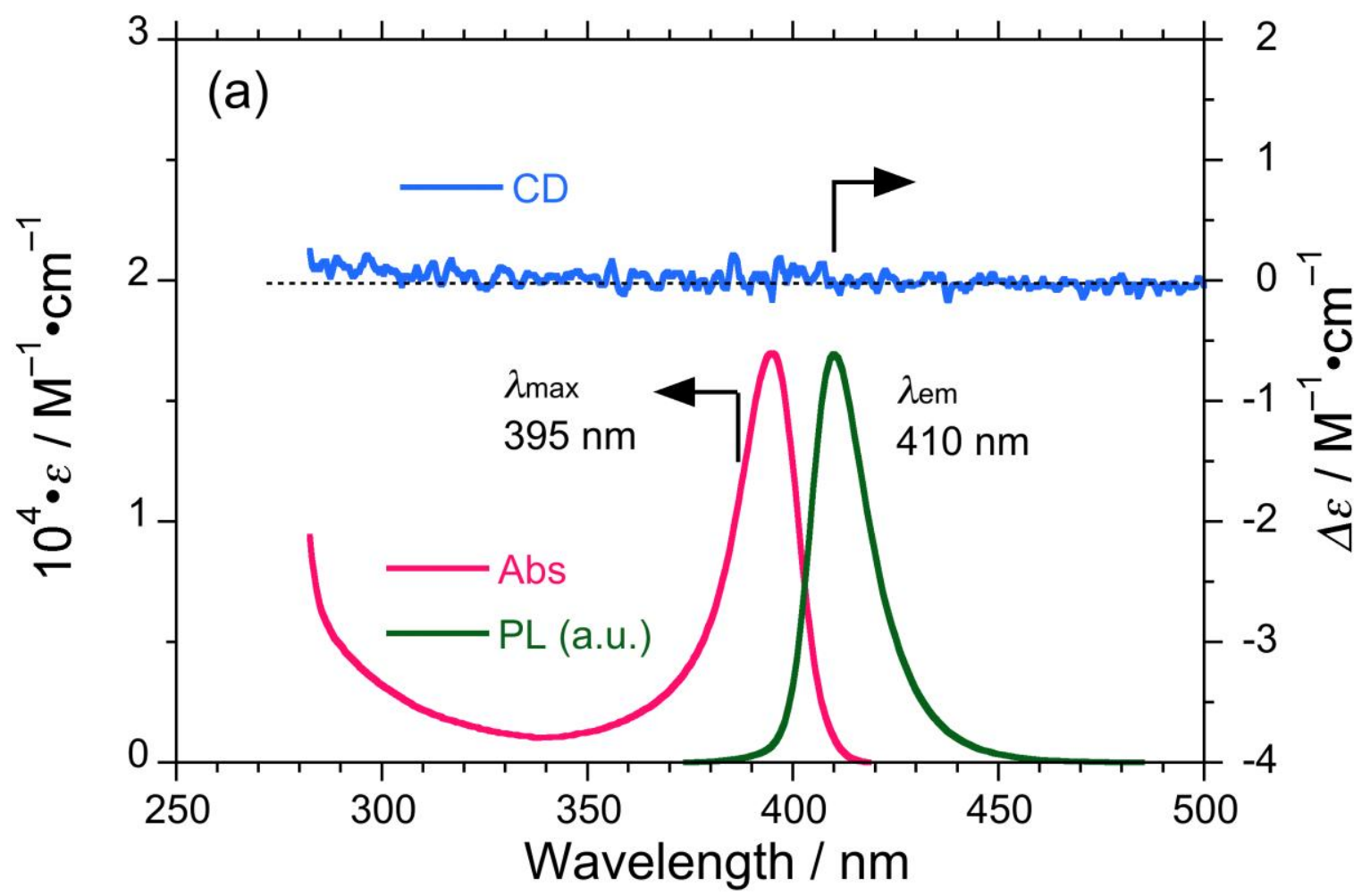




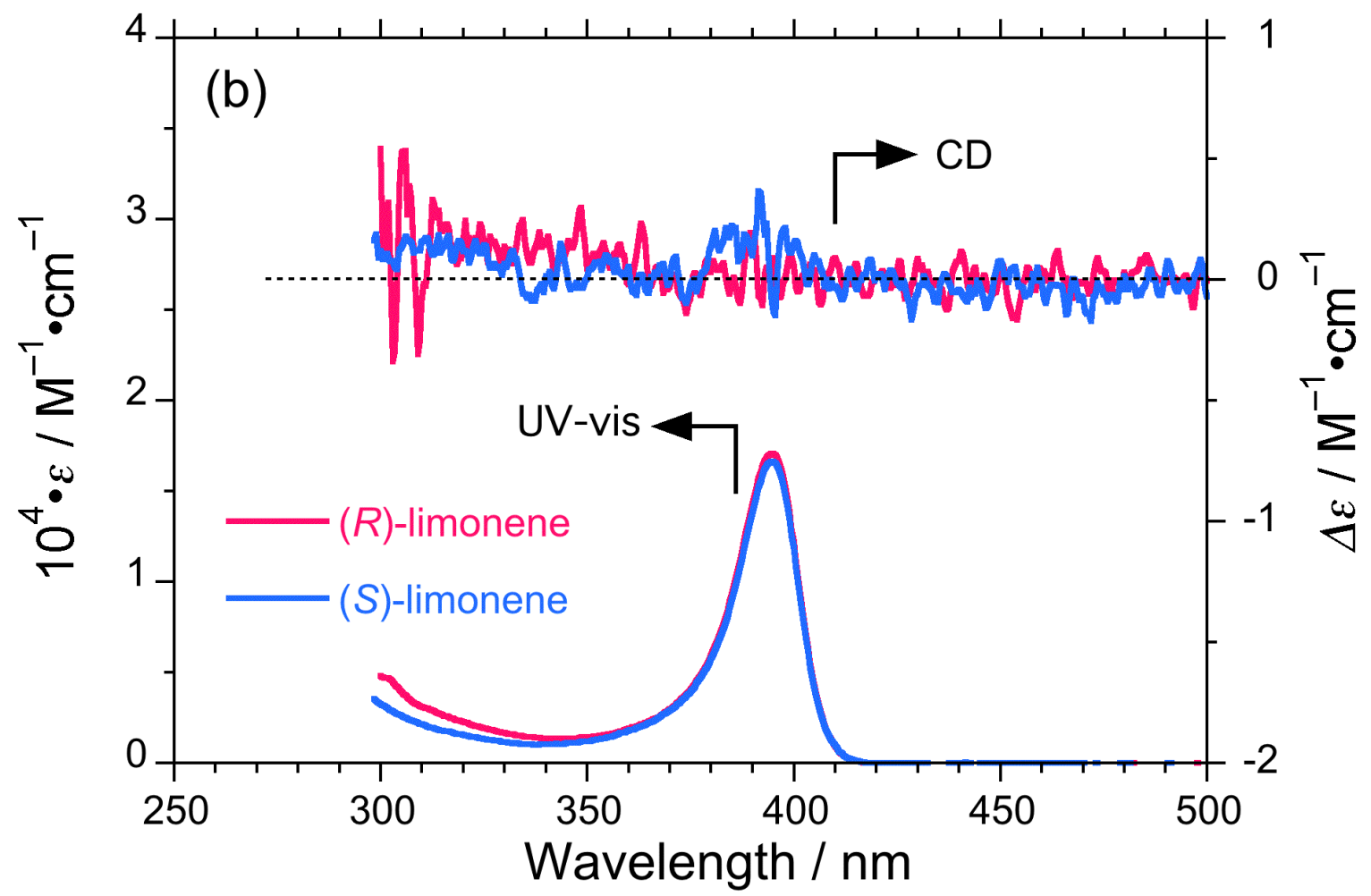

Fig. 3. (a) UV-vis, CD, and PL (excited at $350 \mathrm{~nm}$ ) spectra of $\boldsymbol{n B u P S}$ in $\mathrm{CHCl}_{3}$ solution and (b) UV-vis and CD spectra of $\boldsymbol{n B u P S}$ in $(R)$ - or $(S)$-limonene/ $\mathrm{CHCl}_{3}=0.75 / 2.25(\mathrm{v} / \mathrm{v})$. Path length: $1.0 \mathrm{~cm},[\boldsymbol{n B u P S}]_{0}=1.0 \times 10^{-4} \mathrm{M}^{-1} \mathrm{~cm}^{-1}$.

When $\boldsymbol{n B u P S}$ is dissolved in a mixture of limonene and $\mathrm{CHCl}_{3}(0.75 / 2.25(\mathrm{v} / \mathrm{v}))$, we can see ultra-weak CD signals at $395 \mathrm{~nm}$, due to subtle imbalance between $P$ - and $M-15_{7}$ helices, as shown in Fig. 3b. However, the degree of dissymmetry factor in the CD spectra, $g_{\text {abs }}$ value, is defined as $2 \times\left(A b s_{\mathrm{L}}-A b s_{\mathrm{R}}\right) /\left(A b s_{\mathrm{L}}+A b s_{\mathrm{R}}\right)=\Delta \varepsilon / \varepsilon$, is only $\approx 1 \times 10^{-5}$ for $(S)$-limonene, but not detectable for $(R)$-limonene. The degree of $n$ BuPS $P-M$ imbalance induced by limonene chirality in the good solvents is less than $5 \%$, suggesting that the $\left|g_{\text {abs }}\right|$ value of $7_{3}$-helical dialkylpolysilanes is $\approx 2 \times 10^{-4}$. By adding poor solvent (methanol) to the $\boldsymbol{n B u P S}$ solution, CD- and CPL-active $\boldsymbol{n B u P S}$ is instantly formed during aggregation. This terpene chirality induced aggregation process was already established in our previous papers [44,49-54], however $\boldsymbol{n B u P S}$ and related diarylpolysilanes were not studied.

\subsection{UV-vis and CD characteristics of diarylpolysilane aggregates}

Firstly, the UV-vis and CD spectra of $\boldsymbol{n B u P S}$ aggregates produced in a mixture of $\mathrm{CHCl}_{3}$ and methanol (1.05/1.95 (v/v)) are displayed in Fig. 4a. Any detectable CD signals of $\boldsymbol{n B u P S}$ aggregates are confirmed, though the $\mathrm{Si}-\mathrm{Si}$ exciton band at $391 \mathrm{~nm}(3.155 \mathrm{eV})$ slightly 
blue-shifted relative to the homogeneous solution. The absence of limonene chirality merely induced CD-silent $\boldsymbol{n}$ BuPS aggregates.
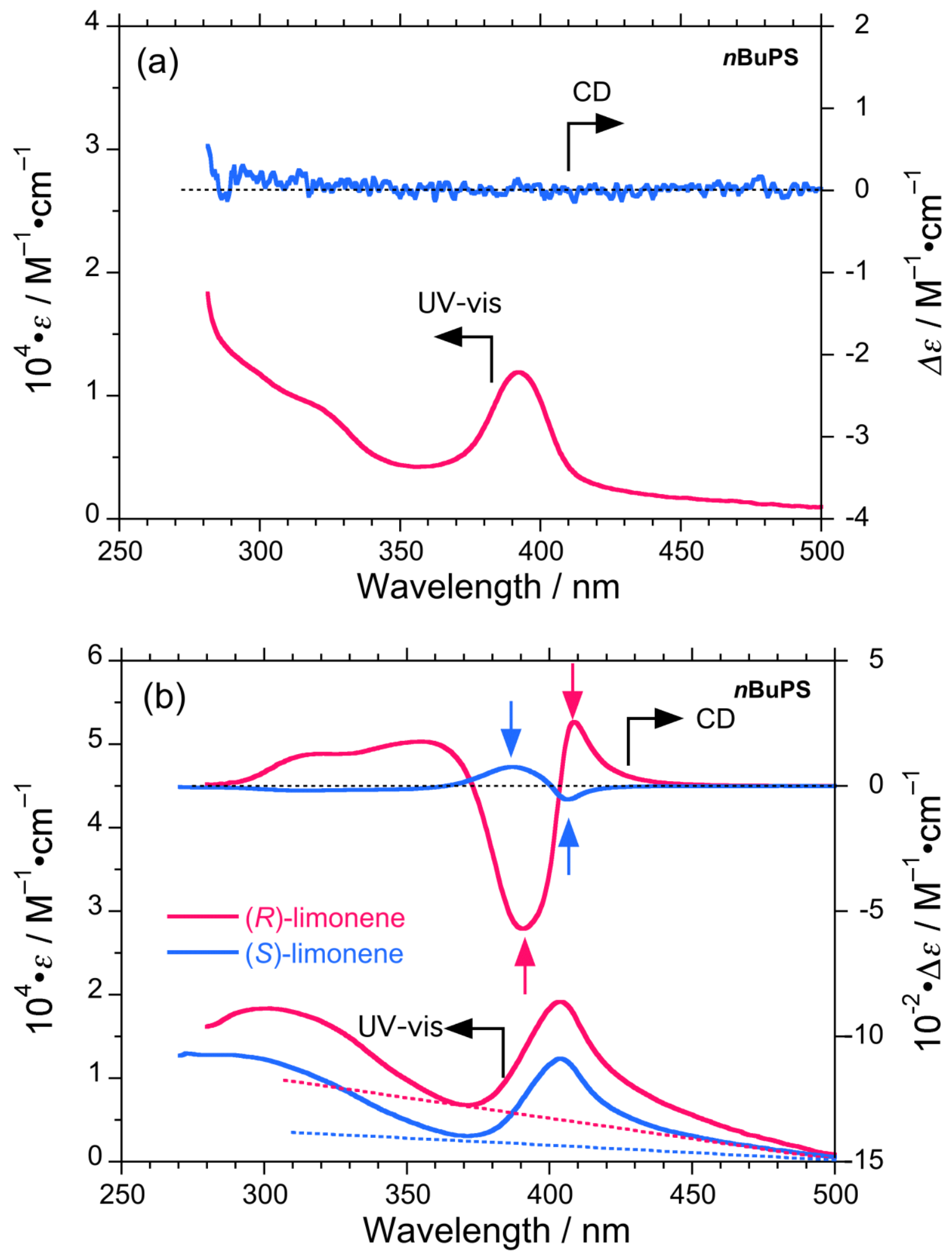

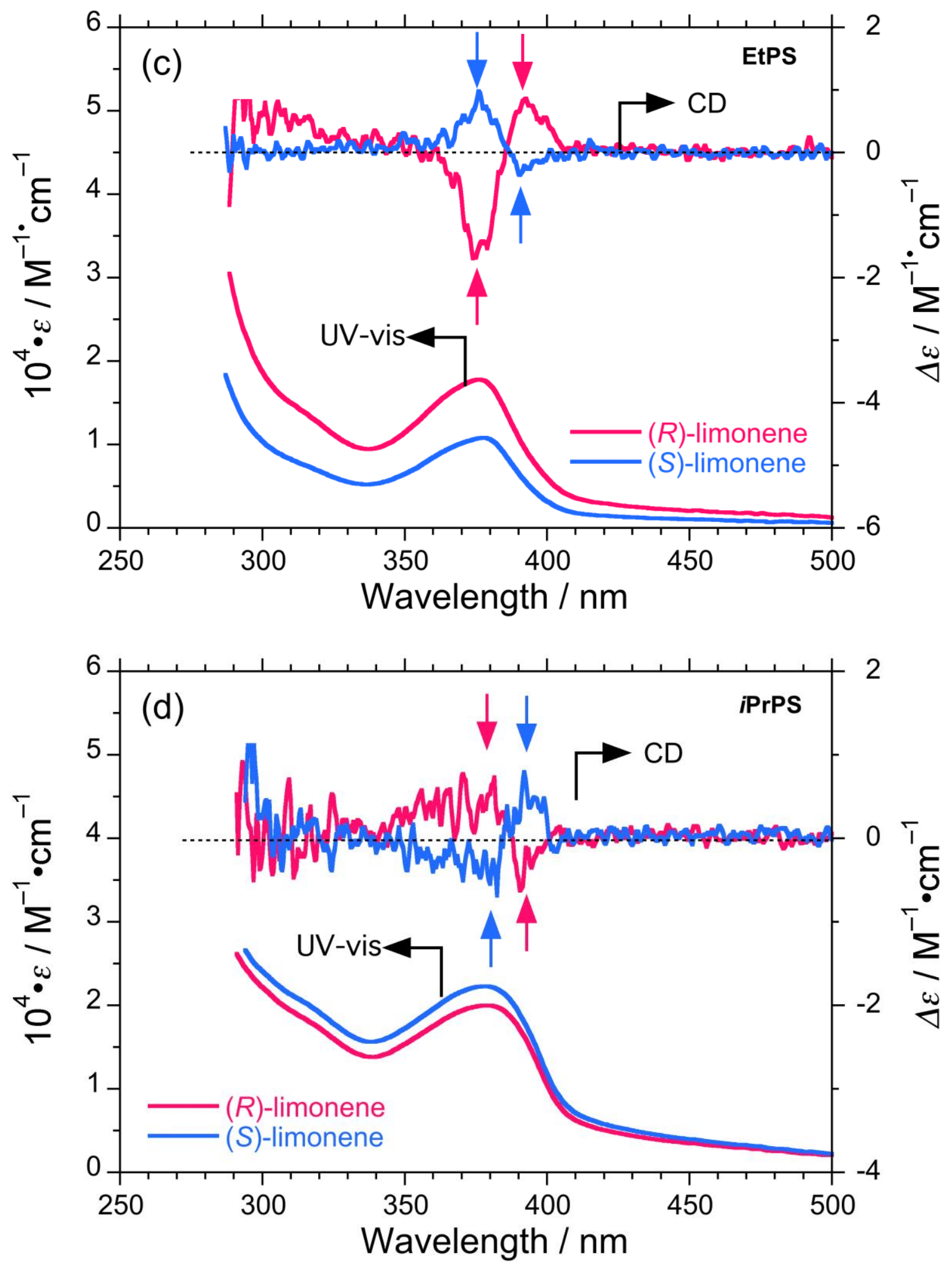

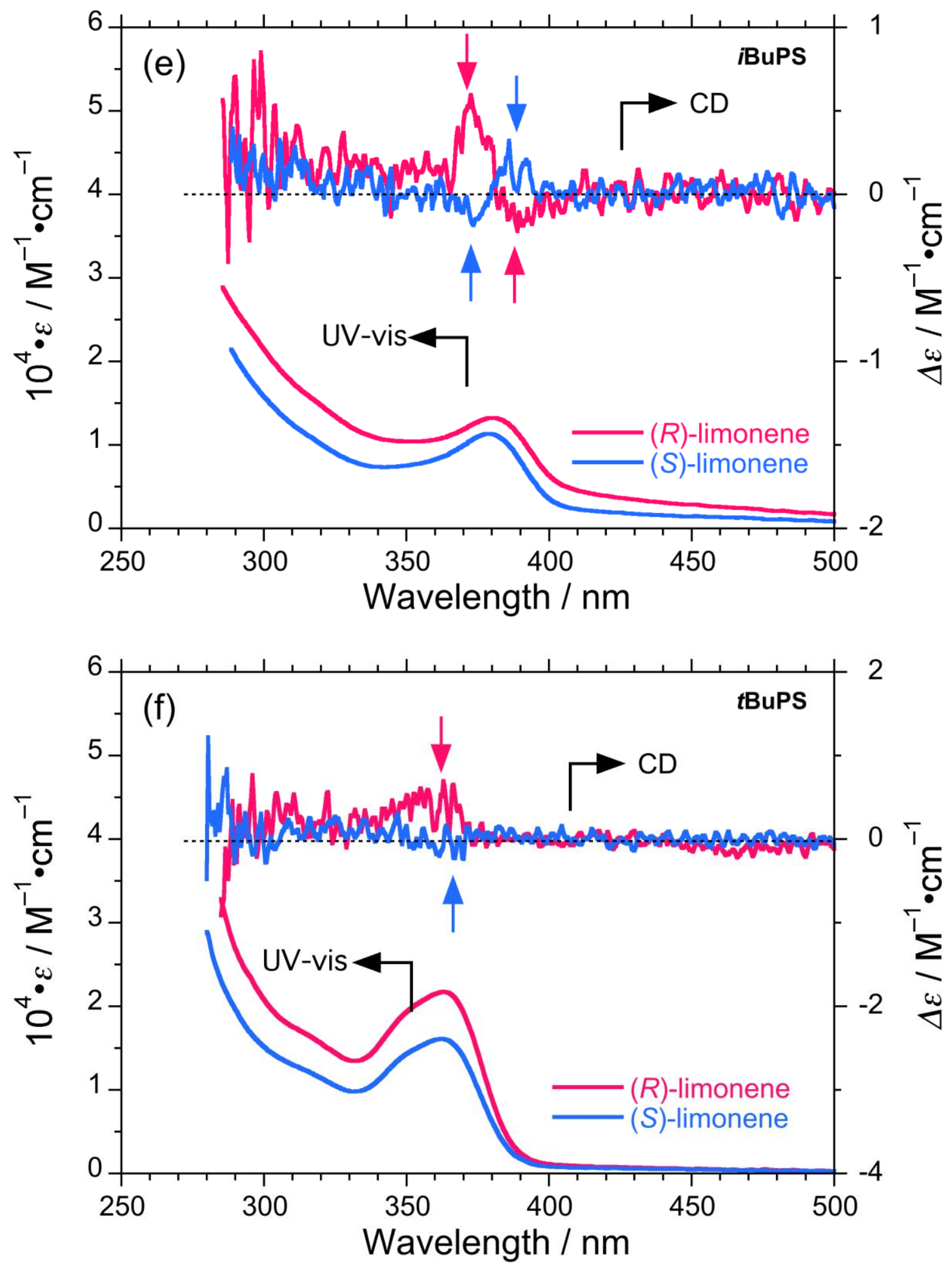

Fig. 4. $C D$ and $U V$-vis spectra of diarylpolysilane aggregates induced by a mixture of limonene, chloroform, and methanol. (a) $\boldsymbol{n B u P S}(0.00 / 1.05 / 1.95(\mathrm{v} / \mathrm{v} / \mathrm{v}))$, (b) $\boldsymbol{n B u P S}$ $(0.75 / 0.30 / 1.95$ (v/v/v)), (c) EtPS (0.75/0.30/1.95 (v/v/v)), (c) $\boldsymbol{i} \boldsymbol{P r P S}(0.40 / 0.30 / 2.30$ (v/v/v)), (d) $i$ BuPS $(0.40 / 0.30 / 2.30(\mathrm{v} / \mathrm{v} / \mathrm{v}))$, (e) $\boldsymbol{i B u P S}(0.40 / 0.30 / 2.30(\mathrm{v} / \mathrm{v} / \mathrm{v}))$, and (f) $\boldsymbol{t} \mathbf{B u P S}$ $(0.40 / 0.30 / 2.30(\mathrm{v} / \mathrm{v} / \mathrm{v}))$. Path length: $1.0 \mathrm{~cm}$, [diarylPS $]_{0}=1.0 \times 10^{-4} \mathrm{M}^{-1} \mathrm{~cm}^{-1}$. 
The chiral solvent including limonene prompted to emerge $\boldsymbol{n B u P S}$ exhibiting gigantic bisignate Cotton bands around $403 \mathrm{~nm}$ during aggregation of $\boldsymbol{n B u P S}$, as given in Fig. $4 \mathrm{~b}$. However, baselines of UV-vis spectra are lifted upward due to Rayleigh scattering of the aggregates, whilst induced CD signals are insensitive to the aggregate scattering.

To evaluate the degree of limonene-chirality induced CD effect, we corrected UV-vis signals with numerically subtracting UV-vis signals by setting UV-vis signals to be zero at $500 \mathrm{~nm}$. The UV-vis signal at $500 \mathrm{~nm}$ does not come from the contribution of the 403-nm absorption tail. Raw data with dotted lines as baseline correction are given in Appendix Fig. S1.

The evaluated $g_{\text {abs }}$ values at the first Cotton bands are $\approx+0.04$ at $408 \mathrm{~nm}$ for $(R)$-limonene and $\approx-0.03$ at $406 \mathrm{~nm}$ for $(S)$-limonene, respectively, whilst the $g_{\text {abs }}$ values at the second Cotton band are $\approx-0.1$ at $391 \mathrm{~nm}$ for $(R)$-limonene and $\approx+0.02$ at $387 \mathrm{~nm}$ for $(S)$-limonene, respectively. The $g_{\text {abs }}(R)$-to- $g_{\text {abs }}(S)$ ratio at the first Cotton band is $\approx 1.3$ in the $S_{0}$ state, while the $g_{\text {abs }}(R)$-to- $g_{\text {abs }}(S)$ ratio at the second Cotton band is $\approx 5$.

The other four diarylpolysilanes (EtPS, $i$ PrPS, $i$ BuPS, and $\boldsymbol{t}$ BuPS) resulted in the corresponding $\mathrm{CD}$-active aggregates in the presence of $(R)$-limonene (or $(S)$-limonene). However, the absolute $g_{\text {abs }}$ values at the first and second Cotton bands significantly reduced by two and three orders of the magnitude and on the order of $10^{-4}$. $\boldsymbol{t}$ BuPS does not show bisignate $\mathrm{CD}$ characteristics and has a single sign weak-CD signals. This weakness arises from weakly entangled aggregation due to lower molecular mass polymers $(4,000-6,000)$, whilst $\boldsymbol{n B u P S}$ has a high molecular mass able to form entangled soft aggregates. Molecular mass may be a critical factor to induce a highly dissymmetrical helical organization in the aggregates, as proven by the enhanced CD signals.

The nature of bisignate couplet CD signals is greatly dependent of the type of alkyl pendants at the para-aryl groups, as summarized in Table 1. $(R)$-chirality of limonene induced the positive couplet signals (positive-sign $\mathrm{CD}$ at the first Cotton and negative-sign $\mathrm{CD}$ at the second band) for diarylpolysilanes with non-branched $n$-butyl and ethyl groups, whilst the $(R)$-chirality oppositely induced the negative couplet signals (negative-sign $\mathrm{CD}$ at the first Cotton and positive-sign $\mathrm{CD}$ at the second band) for diarylpolysilanes with branched alkyl groups, including isopropyl and isobutyl. The choice of non-branched or branched alkyl moiety is another critical factor determining the sign of $\mathrm{CD}$ signals in the aggregates, although for unknown reasons. 
Table 1. Comparisons of Cotton CD sign, $\lambda_{\max }$, and $\lambda_{\mathrm{ex}}$ values of diarylpolysilanes aggregates induced by limonene-containing tersolvent (Data taken from Fig. 4).

\begin{tabular}{|c|c|c|c|c|c|}
\hline & \multirow[b]{2}{*}{$\lambda_{\max }(\mathrm{nm})$} & \multicolumn{2}{|c|}{$\lambda_{\mathrm{ex}}(\mathrm{nm})$} & \multicolumn{2}{|c|}{ CD signs by $(R)$-limonene } \\
\hline & & $\begin{array}{l}\text { 1st Cotton } \\
\text { band }\end{array}$ & $\begin{array}{c}\text { 2nd Cotton } \\
\text { band }\end{array}$ & $\begin{array}{l}\text { 1st Cotton } \\
\text { band }\end{array}$ & $\begin{array}{c}\text { 2nd Cotton } \\
\text { band }\end{array}$ \\
\hline$n B u P S$ & $403(S$ and $R)$ & $407-409$ & $388-391$ & + & - \\
\hline EtPS & $377(S), 378(R)$ & $392-393$ & 376 & + & - \\
\hline „PrPS & $380(S), 383(R)$ & 389 & 372 & - & + \\
\hline IBuPS & $380(S), 382(R)$ & 388 & 372 & - & + \\
\hline$t \mathrm{BuPS}$ & $363(S$ and $R)$ & 376 & & + & \\
\hline
\end{tabular}

\subsection{CD/UV-vis/CPL/PL spectral characteristics of $\boldsymbol{n B u P S}$ aggregates}

Notably, an imbalance in the $g_{\text {abs }}$ values of $\boldsymbol{n B u P S}$ between $(R)$ - and $(S)$-limonene was observed (Fig. 4b) as a matter of fact. A similar but weak limonene chirality tendency in $g_{\text {abs }}$ values can be seen in EtPS, $\boldsymbol{i B u P S}$, and $\boldsymbol{t}$ BuPS aggregates (Figs. 4c-4f). In $\boldsymbol{n B u P S}$ aggregates induced by limonene chirality, mirror symmetry (mirror image relationship in CD spectra) in the ground state is considerably broken, though $(R)$ - and $(S)$-limonene purified in a reduced pressure had an almost identical absolute specific optical rotation $[\alpha]_{\mathrm{D}}$ with the opposite sign in neat limonene. In a series of limonene chirality induced aggregation, we observed that invariably the $\mathrm{CD}$ spectra of $\pi$ - and $\sigma$-conjugated polymers during aggregating with $(R)$ - and (S)-limonene are not an ideal mirror image relationship for reason as yet unsolved [44,49-54]. Similar non-mirror image CD spectra of $\boldsymbol{n B u P S}$ induced by $\alpha$-pinene chirality can be seen in this experiment (Appendix, Figs. S3a-S3d).

CD-signals detect the $S_{0}$-state chirality of substances in the ground state, whilst CPL-signal reflects from the photoexcited $S_{1}$-state chirality with the lowest vibronic mode (Kasha's rule). Importantly, CPL and PL signals do not reflect from the scattering of the aggregates. It should be noted that baselines of CPL and PL are insensitive to the aggregate scattering. Thus, the

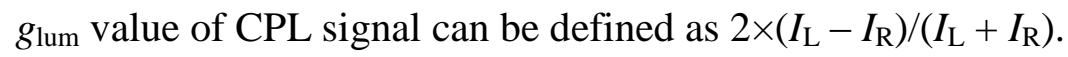



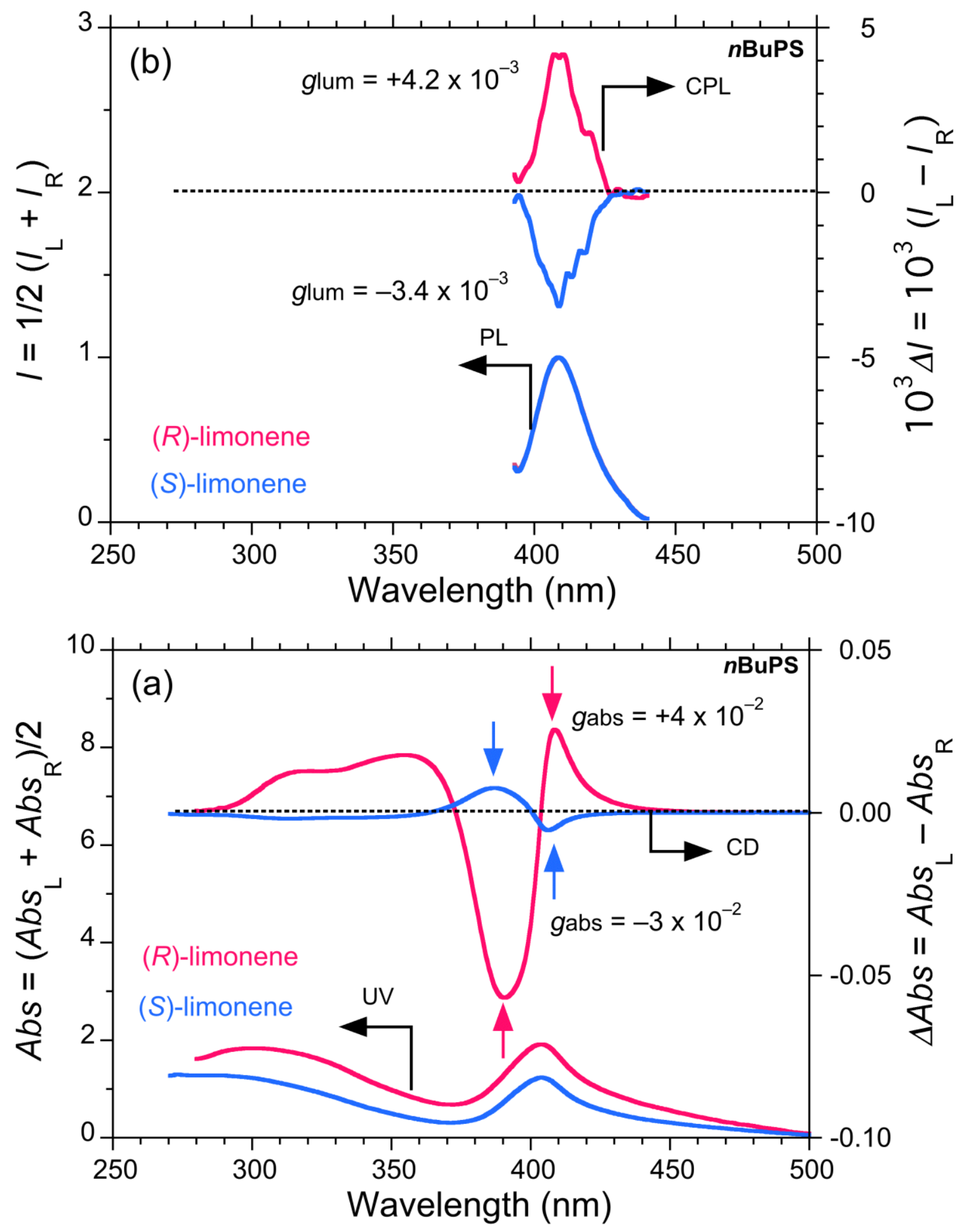
(c)

0

c

0

o

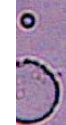

(d)

| 100nini

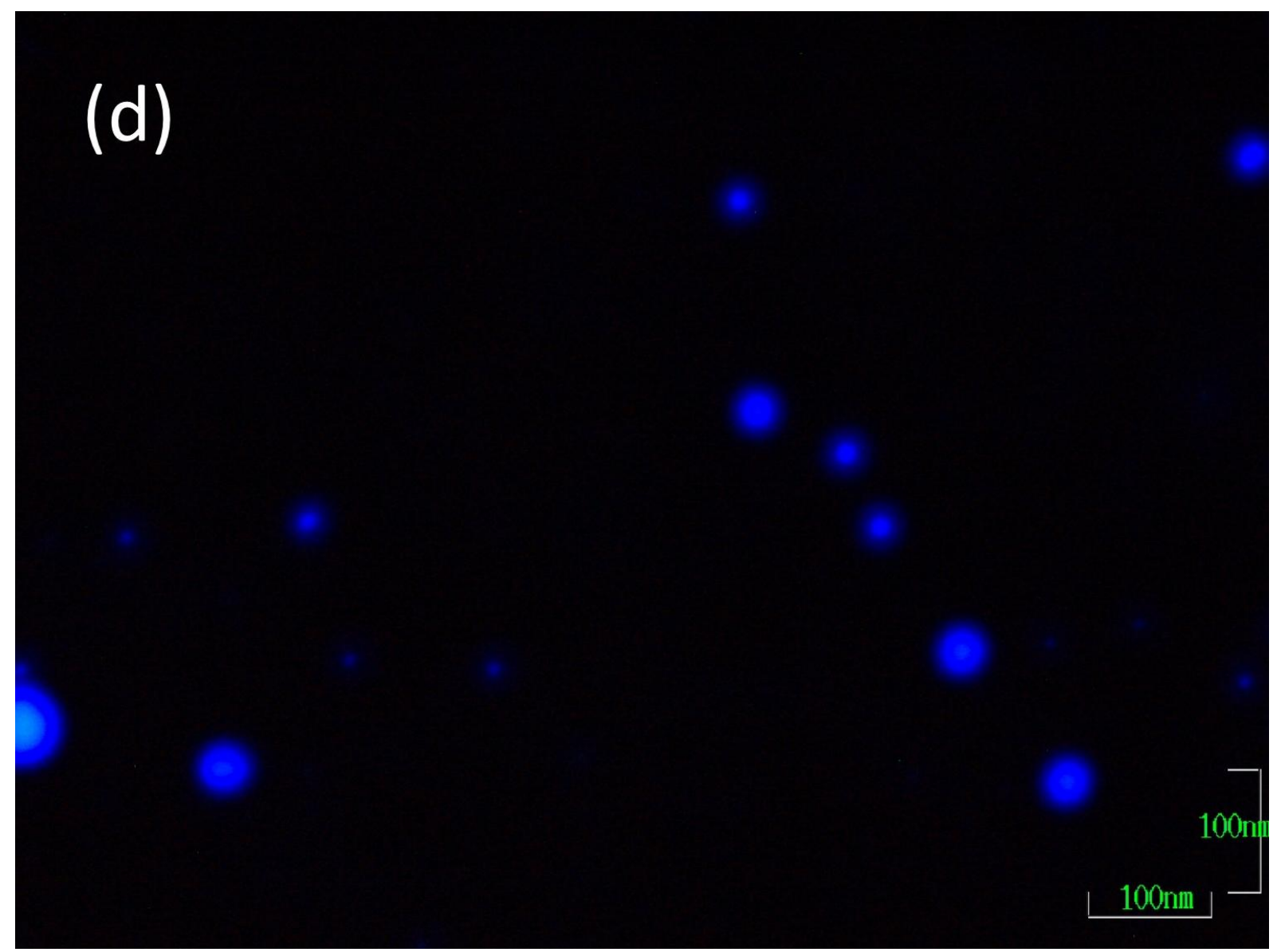


Fig. 5. (a) Normalized UV-vis and CD spectra and (b) PL and CPL spectra of $\boldsymbol{n B u P S}$ aggregates in 0.75/0.30/1.95 (v/v/v)). Non-focused blurred optical microscopic images of $\boldsymbol{n B u P S}$ aggregates produced in $(R)$-limonene/chloroform/methanol $=0.75 / 0.30 / 1.95(\mathrm{v} / \mathrm{v} / \mathrm{v})$ under (c) bright field under white light illumination and (d) its dark field fluorescence images upon $365 \mathrm{~nm}$ excitation (scale bar: $100 \mathrm{~nm}$ ).

The normalized UV-vis and CD spectra of $\boldsymbol{n B u P S}$ aggregates produced in limonene/chloroform/methanol $=0.75 / 0.30 / 1.95(\mathrm{v} / \mathrm{v} / \mathrm{v})$ are shown in Fig. 5a. For comparison, the normalized PL and CPL spectra of the aggregates are shown in Fig. 5b. The dissymmetry factors, $g_{\text {lum }}$, at $410 \mathrm{~nm}$ in the photoexcited state are $+4.2 \times 10^{-3}((R)$-limonene $)$ and $-3.4 \times 10^{-3}((S)$-limonene), respectively. The CPL-sign is identical to that of the CD-sign at the first Cotton band. For example, $(R)$-limonene induces plus-sign $\mathrm{CD}$ and plus-sign CPL, whilst $(S)$-limonene induces minus-sign CD and minus plus-sign CPL.

The magnitudes in $g_{\text {lum }}$ values markedly decreased by $1 / 10$ for $(R)$ - and $(S)$-limonene, compared to the corresponding $g_{\text {abs }}$ values at the first Cotton bands. The $g_{\text {lum }}$ values decreased by $1 / 25$ times for $(R)$-limonene and by $1 / 6$ times for $(S)$-limonene, compared to the $g_{\text {abs }}$ values at the second Cotton bands. The $g_{\text {lum }}(R)$-to- $g_{\text {lum }}(S)$ of $\approx 1.2$ in the $S_{1}$ state is nearly identical to the $g_{\text {abs }}(R)$-to- $g_{\text {abs }}(S)$ ratio at the first Cotton band. The left-right symmetry of the aggregates may be slightly broken in the ground and photoexcited states.

$n$ BuPS aggregates in $(R)$-limonene/chloroform/methanol $(=0.75 / 0.30 / 1.95(\mathrm{v} / \mathrm{v} / \mathrm{v}))$ are visualized by defocused optical microscopic imaging. The bright field image under white light illumination and the corresponding dark field fluorescence images excited at $365 \mathrm{~nm}$ are displayed in Figs. 5c and 5d, respectively. Due to the blurring effect, each aggregate clearly emits a purple-blue color, though the actual size of the aggregates is smaller than $1.0 \mu \mathrm{m}$, as mentioned in a later section. Under non-blurring condition, each $\boldsymbol{n B u P S}$ aggregates clearly emits as very small blue color dots (Fig. S2). Possibly, each dot weakly emits the CPL signal because of the CPL-active $\boldsymbol{n B u P S}$ aggregates.

\subsection{The $g_{\text {abs }}$ value of $\boldsymbol{n B u P S}$ aggregates vs $(R)$ - and $(S)$-limonene fractions in tersolvents}

Recent knowledge and understanding of the conformational structure indicated that $(R)$-limonene mainly exists as a mixture of three stable rotational isomers with the quasi-equatorial orientation of the isopropenyl group on the cyclohexene ring [62,63]. Minor rotational isomers are three unstable isomers with the quasi-axial orientation of the isopropenyl group on the cyclohexene ring [62]. Although vibrational circular dichroism 
(VCD) experiment followed by population analysis of neat $(S)$-limonene was not reported by the same research groups $[62,63]$, these results prompted us to investigate the $g_{\text {abs }}$ value of $n$ BuPS aggregates in detail.
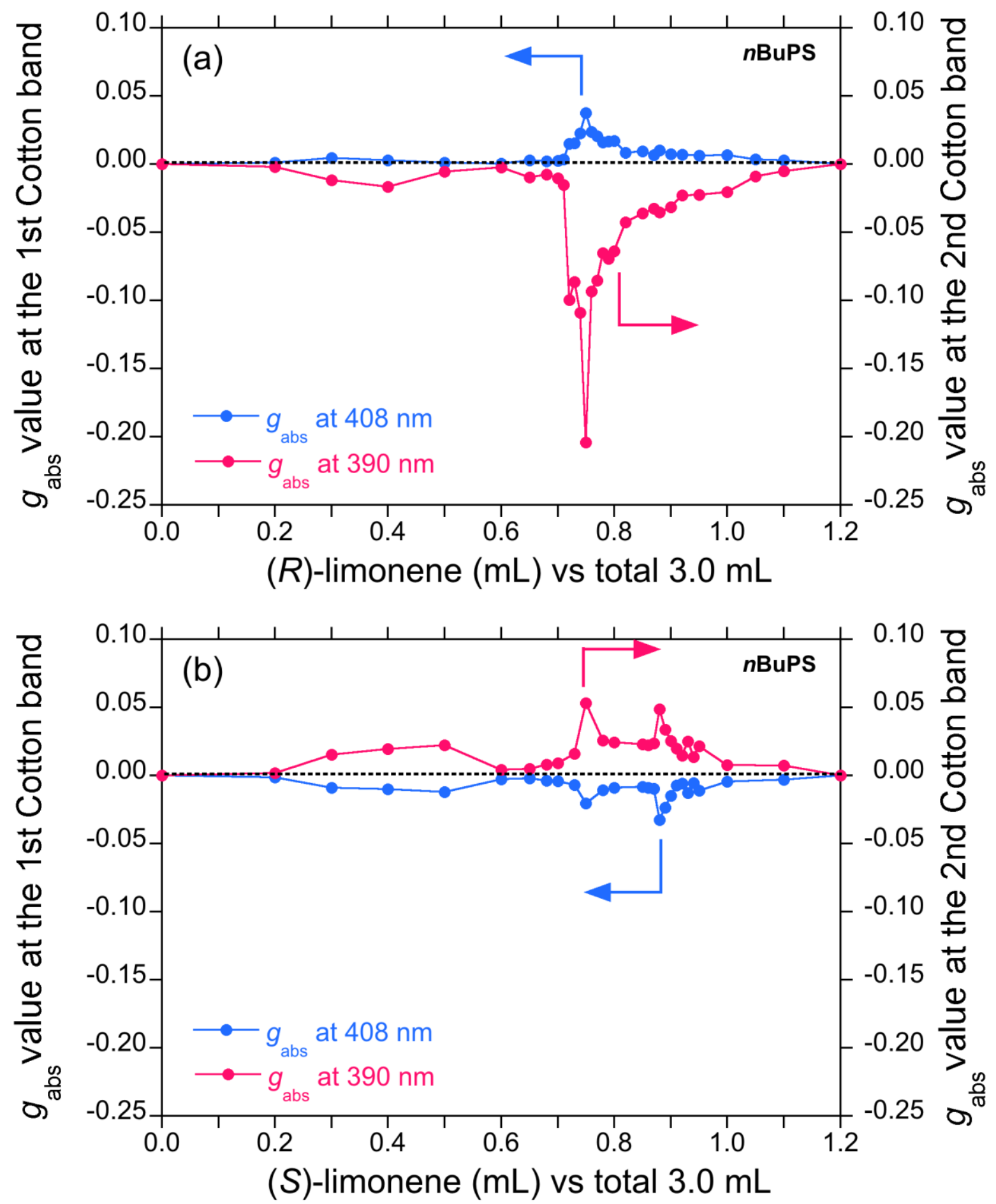

Fig. 6. The $g_{\text {abs }}$ values at the first and second Cotton bands of $\boldsymbol{p B u P S}$ aggregates as a function of volume fraction of $(S)$ - or $(R)-\square \square \square \square \square \square \square \square$ in a mixture of $\square \square \square \square \square \square \square \square$, chloroform, 
and methanol. (a) (R)- $\begin{gathered}\text { a } \\ \text { a }\end{gathered}$ total volume $3.0 \mathrm{~mL}$.

The $g_{\text {abs }}$ values at the first and second Cotton bands of $\boldsymbol{n B u P S}$ aggregates as a function of volume fraction of $(R)-\square \square \square \square \square \square \square \square$ in a mixture of $(R)-\square \square \square \square \square \square \square \square$, chloroform, and methanol are given in Fig. 6a. Similarly, the $g_{\text {abs }}$ values of $\boldsymbol{n B u P S}$ aggregates as a function of volume fraction of (S)- $\square \square \square \square \square \square \square \square$ in a mixture of (S)- $\square \square \square \square \square \square \square \square$, chloroform, and methanol are given in Fig. 6b. Herein, chloroform is fixed to $0.3 \mathrm{~mL}$ and a total volume is 3.0 mL. From Fig. 6a, it is evident that the $g_{\text {abs }}$ value resonantly enhances at a specific volume fraction of $(R)$-limonene $(0.75 \mathrm{~mL}, 25 \%$ in volume $)$, but weakly magnifies at a $0.40-0.50 \mathrm{~mL}$ of $(R)$-limonene (13-17 \% in volume) and at $0.90-1.0 \mathrm{~mL}$ of $(R)$-limonene $(30-35 \%$ in volume). The $g_{\text {abs }}$ value at the second Cotton band attains $\approx-0.1$ ( $25 \%$ in volume), equivalent to $5 \%$ of circular polarization. On the other hand, from Fig. $6 \mathrm{~b}$, the $g_{\text {abs }}$ value resonantly magnified at three specific volume fractions when ca. $0.50 \mathrm{~mL}(17 \%$ in volume $), 0.75 \mathrm{~mL}$ ( $25 \%$ in volume), and $0.88 \mathrm{~mL}$ ( $29 \%$ in volume) of $(S)$-limonene.

These specific two and/or three volume fractions of limonene as a matter of fact may have a special meaning and be interpreted with the three possible conformational isomers of limonene in the mixed solution. The rotation of the isopropenyl group in limonene is responsible for resonantly magnified $g_{\text {abs }}$ value of $\boldsymbol{n B u P S}$ aggregates at the specific volume fractions.

According to a recent work [63], the magnitude and sign of $[\alpha]_{\mathrm{D}}$ and VCD signals depend on the dihedral angle (C9-C8-C4-H4) of isopropenyl group on the quasi-equatorial cyclohexene ring [63]. The optimized dihedral angles of isopropenyl group were $+129.52^{\circ}$ (conformer 1, methyl moiety of isopropenyl is in-plane to cyclohexenyl ring), $-138.75^{\circ}$ (conformer 2, methyl moiety of isopropenyl is out-of-plane and up-geometry to the ring) and $-8.03^{\circ}$ (conformer 3 , methyl moiety of isopropenyl is out-of-plane and down-geometry to the ring). The populations of conformers 1, 2, and 3 were estimated to 39:31:30, respectively. The calculated $[\alpha]_{\mathrm{D}}$ values were $+430^{\circ}$ (conformers 1), $-140^{\circ}$ (conformers 2), and $-45^{\circ}$ (conformers 3), respectively. Similarly, the calculated signs of VCD signals at $1720 \mathrm{~cm}^{-1}$ due to isopropenyl $v_{\mathrm{C}=\mathrm{C}}$ bond had very intense (+)-sign (conformers 1 ), very intense (-)-sign (conformers 2), and intense (+)-sign (conformers 3), respectively. As a result, optical rotation dispersion (ORD) and CD spectra of $(R)$-limonene in solution greatly depends on the population of three conformers in addition to nature of the solvent. 
If the populations of three conformers are susceptibly changeable by the polarity of a mixture of limonene, methanol, and chloroform, certain conformers of $(R)$ - and $(S)$-limonene may be enriched within the aggregates at these specific volume fractions. These results imply that a specific conformation among six possible isomers of $(R)$ - and $(S)$-limonene efficiently induces the $\boldsymbol{n B u P S}$ aggregates leading greatly magnified CD signals. For example, one of three equatorial $(R)$-limonene conformers selectively interacts with $\boldsymbol{n B u P S}$, whilst all three equatorial ( $S$ )-limonene conformers equally interact with $\boldsymbol{n B u P S}$. It is noted that the $\left|g_{\text {abs }}\right|$ value at the second Cotton band of $(R)$ - and $(S)$-limonene differs by 5 times, as mentioned above. $(R)$-limonene more efficiently tends to induce CD-signal to $\boldsymbol{n B u P S}$ aggregates than $(S)$-limonene, due to as yet unresolved reasons.

We verified the reproducibility of the anomaly, particularly, around the resonantly enhanced volume fractions of $(R)$ - and $(S)$-limonene by employing multiple experiments in different days and weeks. Typically, it took at least two days to obtain a series of CD/UV-vis measurements for $(R)$-limonene ranging from 0.0 to $1.2 \mathrm{~mL}$ associated with $0.01-0.02 \mathrm{~mL}$ interval, followed by a series of $(S)$-limonene chirality induced experiments. It thus needed several days to yield several sets of $(R)$ - and $(S)$-limonene induced aggregation experiments. Similarly, we applied this protocol to $(1 R)$ - and $(1 S)$-pinene chirality induced aggregation experiments.

The refractive index at $656 \mathrm{~nm}\left(n_{\mathrm{c}}\right)$ and solution viscosity $(\eta)$ as a function of volume fraction of $(S)$ - and $(R)$-limonene in a mixed solvent are plotted in Appendix, Fig. S1d. The values of $\eta$ and $n_{\mathrm{c}}$ increased almost linearly with an increase of limonene fraction regardless of $(S)$ - and $(R)$-chirality. However, the $n_{\mathrm{c}}$ value slightly deviated to a lower value, as indicated by the red arrow, when $(R)$-limonene was $0.85 \mathrm{~mL}$ ( $28 \%$ in volume). This subtle alteration may be interpreted with the enhanced $g_{\text {abs }}$ value of $\boldsymbol{n B u P S}$ at $(R)$-limonene around $0.75 \mathrm{~mL}$.

A possible scenario for this anomaly may be ascribed to certain impurities included in distilled limonene. Previously, we characterized the enantiopurity of the distilled limonenes using chiral GC column (Supelco $\beta$-DEX-120) as well as optical rotation at $589 \mathrm{~nm}$ [49]. The results were $(R)$-limonene $>99.1 \%$ ee and $[\alpha]^{27}+101.55^{\circ}$ (neat) and $(S)$-limonene $>99.3 \%$ ee and $[\alpha]^{27}+103.19^{\circ}$ (neat). At the present stage, we do not think that the subtle differences in enantiopurity and $[\alpha]^{27}$ values between $(R)$ - and $(S)$-limonene are responsible for the this anomaly.

Other reason arises from an inherent left-right asymmetry between $(R)$ - and $(S)$-limonene. Noting that $(R)$-limonene is a naturally abundant terpene, while $(S)$-limonene is not. Similar 
$(R)-(S)$ asymmetry chirality inducibility can be invariably observed in several polymer aggregations $[39,44-47,49-54]$. We infer briefly that this limonene chirality anomaly may arise from universal origin, as discussed in section 2.8 .

Similar to monocyclic limonene, bicyclic $\alpha$-pinene can induce couplet-like CD signals in $\boldsymbol{n B u P S}$ aggregates. Raw CD and UV-vis spectra and their normalized $\boldsymbol{n B u P S}$ CD and UV-vis spectra produced in a mixture of $\alpha \square$ pinene, chloroform, and methanol $(0.65 / 0.30 / 2.05$ (v/v/v)) are given in Appendix, Figs. S3a-S3b. Evidently, the $g_{\text {abs }}$ values at the second Cotton band attain $-2 \times 10^{-2}$ for $(1 R)$-pinene and $+2 \times 10^{-2}$ for $(1 S)$-pinene, although the $\lambda$ value at extremum wavelength $\left(\lambda_{\text {ext }}\right)$ in CD spectrum and $\lambda_{\max }$ in UV-vis spectrum are subtly different. The $\lambda_{\text {ext }}$ values are located at $402 \mathrm{~nm}$ and $386 \mathrm{~nm}$ for (1R)-pinene, while they are $406 \mathrm{~nm}$ and $386 \mathrm{~nm}$ for (1S)-pinene. The $\lambda_{\max }$ values of $(1 R)$ - and (1S)- $\alpha$-pinene are $399 \mathrm{~nm}$ and $404 \mathrm{~nm}$, respectively. These deviations in $\lambda_{\text {ext }}$ and $\lambda_{\max }$ values were reproducible by multiple experiments.

Similar to the case of limonene, the $g_{\text {abs }}$ values of $\alpha$-pinene-induced $\boldsymbol{n B u P S}$ aggregates depend somewhat on the volume fraction of $\alpha$-pinene (Appendix, Figs. S3c-S3d). Although this tendency is not obvious compared to the case of limonene, there exist optimized volume fractions of $\alpha$-pinene to efficiently induce the magnified CD-active $n$ BuPS aggregates. Differences in inducibility of molecular chirality between limonene and $\alpha$-pinene to $\boldsymbol{n B u P S}$ may come from the adaptability of terpene; limonene adopts a floppy monocyclic framework allowing three stable and three metastable conformations, though $\alpha$-pinene is in a rigid bicyclic framework with one stable conformation.

At the moment, we cannot show up direct evidence that a certain conformer among three conformers of $(R)$ - and $(S)$-limonene, depending on their solvent fractions, is specifically interacted with the diarylpolysilane aggregates. A previous pape showed the marked $(R)$ - and $(S)$-limonene volume fraction dependence including amplifications and multiple inversions in $g_{\text {abs }}$ characteistics of three rodlike dialkylpolysilane aggregates [50]. We assume that a floppy monocyclic terpene, limonene, is one of the most adaptable chiral substances susciptible to the natures of the surrounding solvents and rodlike polysilanes.

\subsection{Alteration of aggregation size of $\boldsymbol{n B u P S}$ with aging time}

Tempo-spatial characteristics of aggregate size and its distribution in the $\boldsymbol{n B u P S}$ aggregates were examined by DLS technique at four different aging times $(5,15,30$, and $60 \mathrm{~min})$ after evolution ( $<1 \mathrm{~min}$ ) of the aggregates (Figs. 7a-7b). CD/UV-vis spectra measurements of the fresh aggregate sample were completed within $3 \mathrm{~min}$. However, UV-vis measurements of 
prolonged aggregate specimens were difficult due to a high turbidity. Time-dependent alteration in the aggregate size was detectable only by DLS technique. Because of the open-access condition between interior and exterior of the aggregates surrounded by the chiral and achiral constitutes, we can understand how the aggregate propagates with aging time. All raw data are displayed in Appendix, Figs. S4 and S5.

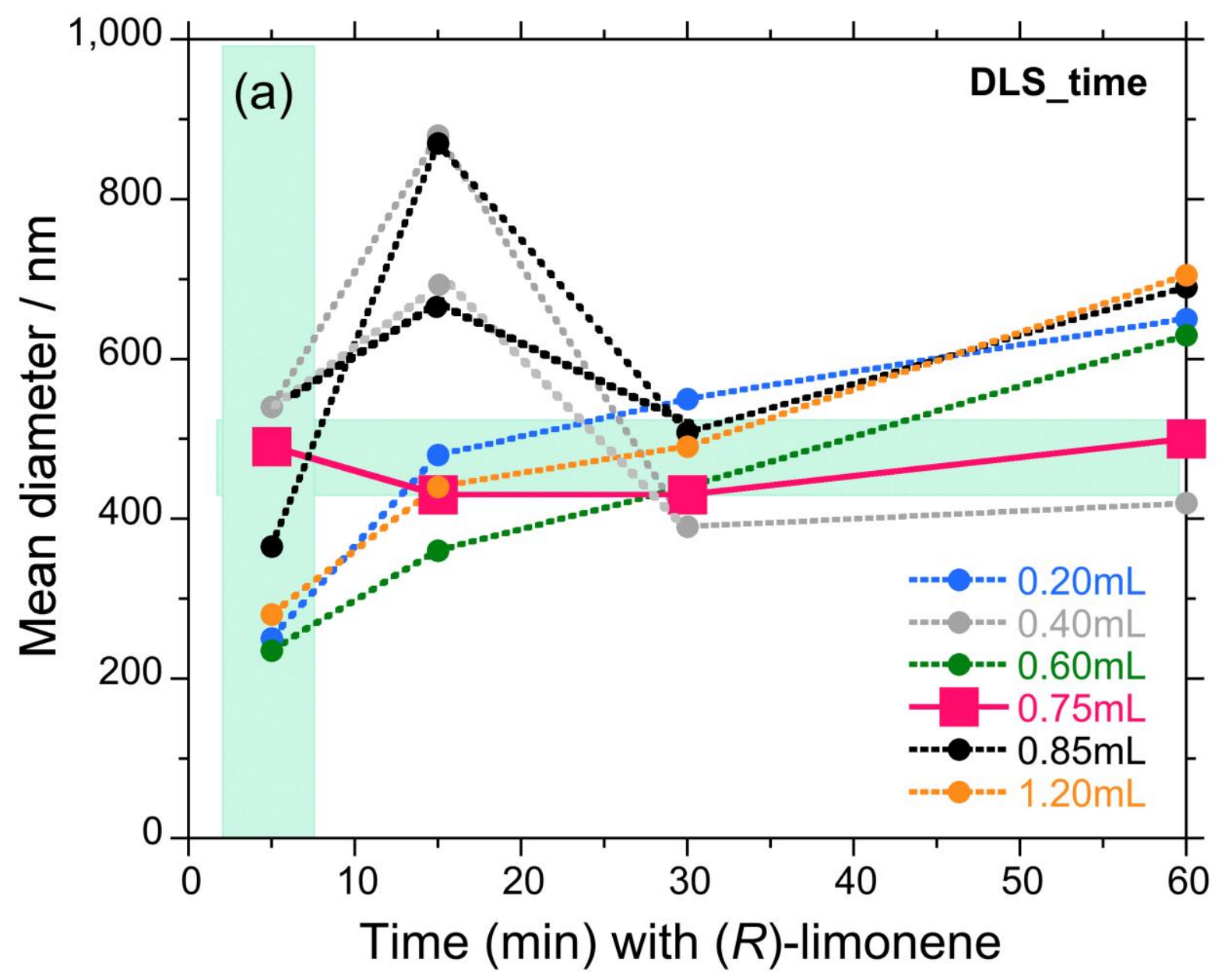




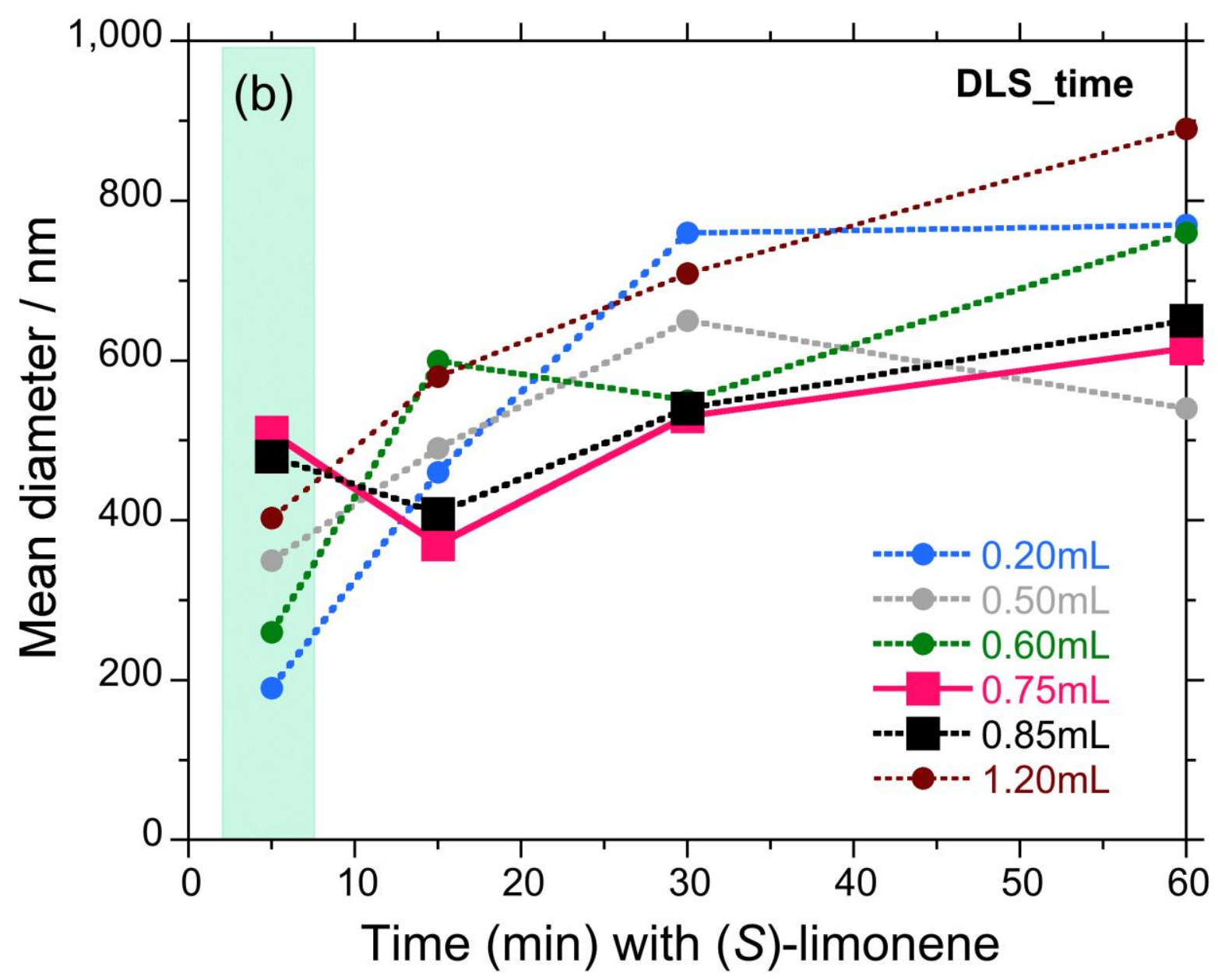

Fig. 7. Alteration in aggregate sizes of $\boldsymbol{n B u P S}$ aggregates generated in various $\square \square \square \square \square \square \square$, chloroform, and methanol fractions revealed by dynamic light scattering (DLS). (a) $(R)$-limonene. chloroform $=0.3 \mathrm{~mL}$, total volume $3.0 \mathrm{~mL}$. (b) $(S)$-limonene. chloroform $=0.3$ $\mathrm{mL}$, total volume $3.0 \mathrm{~mL}$.

As evident from Figs. 7a-7b and Figs. S4-S5, initial size (at 5 min aging) of the aggregates ranged from 250 and $550 \mathrm{~nm}$ for $(R)$-limonene and from 200 and $500 \mathrm{~nm}$ for $(S)$-limonene. Larger sizes were initially formed when $0.75 \mathrm{~mL}$ and $0.40 \mathrm{~mL}$ of $(R)$-limonene were used. Similarly, larger sizes were initially formed when $0.75 \mathrm{~mL}$ and $0.85 \mathrm{~mL}$ of $(S)$-limonene are employed.

In aggregates produced in $0.20,0.60$, and $1.20 \mathrm{~mL}$ of $(R)$-limonene, the size increased with time, approaching a level-off value at $400-700 \mathrm{~nm}$. Aggregates in $0.40 \mathrm{~mL}$ and $0.85 \mathrm{~mL}$ of $(R)$-limonene abruptly collapsed to two sizes (700 and $900 \mathrm{~nm}$ ) at $15 \mathrm{~min}$, then, approached a smaller level-off size of $\approx 400 \mathrm{~nm}$ and $\approx 700 \mathrm{~nm}$, respectively. Exceptionally, the aggregate size produced in $0.75 \mathrm{~mL}$ of $(R)$-limonene existed stably at least for $60 \mathrm{~min}$, and was kept in its initial size of $\approx 450-500 \mathrm{~nm}$. The $0.75 \mathrm{~mL}-(R)$-limonene induced the large $g_{\text {abs }}$ value among limonene containing solvents tested in this work (Fig. 6a). 
In aggregates produced in $0.20,0.50,0.60$, and $1.20 \mathrm{~mL}$ of $(S)$-limonene, the size monotonically increased with aging time, approaching a level-off value of $\approx 550-900 \mathrm{~nm}$. Aggregates produced in 0.75 and $0.85 \mathrm{~mL}$ of $(S)$-limonene tended to fluctuate between 350 and $650 \mathrm{~nm}$. The 0.75 and $0.85 \mathrm{~mL}$ of $(S)$-limonene afforded considerably enhanced $g_{\text {abs }}$ values to $\boldsymbol{n B u P S}$ aggregates (Fig. 6b).

These DLS data were reproducible within deviation of $\pm 10-20 \mathrm{~nm}$ in aggregate size based on three independent measurements. It is thus assumed that tempo-spatial stability in the aggregate sizes is interpreted to the $g_{\text {abs }}$ values of $n$ BuPS during the aggregation (Figs. $6 \mathrm{a}-6 \mathrm{~b})$. The long-term stability of the aggregates in $0.75 \mathrm{~mL}$ of $(R)$-limonene afforded the greatest $g_{\text {abs }}$ value, while the fluctuating aggregates in the 0.75 and $0.85 \mathrm{~mL}$ of $(S)$-limonene led to better $g_{\text {abs }}$ values. Other unstable aggregates had weaker $g_{\text {abs }}$ values. When compared to the level-off value of aggregate sizes at $60 \mathrm{~min},(S)$-limonene induced larger sizes in a range of 550 and $900 \mathrm{~nm}$, while $(R)$-limonene tended to afford rather smaller sizes in a range of 400 and $700 \mathrm{~nm}$.

\subsection{Alteration in aggregation size of EtPS, $\boldsymbol{i P r P S}, \boldsymbol{i B u P S}$ and $\boldsymbol{t}$ BuPS with aging time}

DLS measurements at two aging times ( 5 and $30 \mathrm{~min}$ ) were conducted for aggregates of EtPS, $i$ PrPS, $i$ BuPS and $\boldsymbol{t}$ BuPS (Appendix, Fig. S6). In this case, a volume ratio between limonene, $\mathrm{CHCl}_{3}$, and methanol was fixed to $0.40 / 0.30 / 2.30(\mathrm{v} / \mathrm{v} / \mathrm{v})$ for $\boldsymbol{i} \mathbf{P r P S}, \boldsymbol{i B u P S}$ and tBuPS aggregates, while 0.75/0.30/1.95 (v/v/v) for EtPS. Although no significant differences in mean aggregate size between $(S)$ - and $(R)$-limonene for EtPS and $\boldsymbol{i B u P S}$ at aging times (5 $\min$ and $30 \mathrm{~min}$ ) were observed, noticeable differences between $(S)$ - and $(R)$-limonene (5 min and $30 \mathrm{~min})$ for $\boldsymbol{i}$ PrPS and $\boldsymbol{t}$ BuPS can be seen. EtPS $((S)$-limonene), EtPS $((R)$-limonene), $\boldsymbol{i}$ PrPS $((R)$-limonene), $\boldsymbol{t}$ BuPS $((R)$-limonene), and $\boldsymbol{t}$ BuPS $((S)$-limonene) had mean aggregate size of $\approx 700-900 \mathrm{~nm}$ (5 min), while $\boldsymbol{i P r P S}((S)$-limonene), $\boldsymbol{i B u P S}((S)$ - and $(R)$-limonene) had rather smaller size of $\approx 150-200 \mathrm{~nm}$. Moreover, $\boldsymbol{i P r P S}((R)$-limonene $)$ and $\boldsymbol{t} \mathbf{B u P S}((R)-$ and $(S)$-limonene) at $30 \mathrm{~min}$ tend to further grow their aggregate size, compared to those at $5 \mathrm{~min}$.

\subsection{Comments on the subtle differences in the limonene chirality induced aggregation}

The origin of the limonene-chirality dependent stability of the aggregate size is unresolved. However, subtle differences in $L-D(R-S$ or $P-M)$ preferences of chiral bioresources including limonene, amino acids, oligopeptides, and polymers under fluidic conditions could be enriched into an interior of colloidal aggregates, followed by the measurable values in their sizes [64-67]. 
A possibility of $L-D$ preference at molecular level has been long discussed by several researchers [64-73] in terms of hierarchical scenarios due to inherent handedness ubiquitously existing in our universe, for example, handed weak neutral currents and anti-neutrinos [14,15,64-75]. Elemental particle physicists, astrophysicists, and molecular physicists assume that $C P$ (charge-parity) symmetry breaking, as proven by recent $B^{0}$-meson decay experiments at SLAC (USA) and KEK (Japan), should be responsible for dominance of handed chiral matters over their anti-matters in our universe [76-80].

Previously, Hegstrom et al. theoretically predicted the existence of an ultratiny energy inequality (so-called PVED) between left- and right-twisted ethylene on the order of $4 \times 10^{-20}$ Hartree $\left(\approx 2 \times 10^{-17} \mathrm{kcal} \mathrm{mol}^{-1}\right)$ due to parity violation at closed-shell molecular level $[68,69]$. A possibility of whether the tiny inequality is detectable has been long argued since 1960s $[15,71]$. If sterically distorted isopropenyl group and/or cyclohexenyl ring were regarded as models of the hypothetical twisted ethylene, the subtle energy inequality could be enhanced as detectable level of CD/CPL signals in liquid phase during the aggregation associated with solvent quantity of isomeric limonene conformations. Noticeable differences between $(S)$ - and $(R)$-limonene in diarylpolysilane aggregates as chiroptical probes could infer the consequence of the realistic models if handed, odd-parity weak neutral current exists. It is thus awaited further comparative studies between $(S)$ - and $(R)$-limonene in liquid phases by means of $\mathrm{CD}$, ORD, VCD, Raman optical activity, NMR, and computational calculations if the same and other research groups can verify our radical speculations [62,63].

\section{Conclusions}

Aiming at realizing an artificial polymeric model of an open-flow coacervate system in the ground and photoexcited states, we designed soft-matter aggregates surrounded by non-polar terpene and achiral solvents. The aggregates were made of photoluminescent bis(p-n-butylphenyl)polysilanes (nBuPS) which is a non-charged stiff CD-silent and CPL-silent polymer with high molecular mass $\left(M_{\mathrm{w}}=8.49 \times 10^{4}, M_{\mathrm{n}}=2.93 \times 10^{4}\right)$. $(S)$ - and $(R)$-limonene acted as efficient stereogenic center transferable solvents during aggregation, though $(1 S)$ - and $(1 R)$-pinene were also efficient. The behavior of solvent chirality induced aggregation was characterized by $\mathrm{CD}$ and CPL spectroscopic data analysis. The $\boldsymbol{n B u P S}$ aggregates generated in a mixture of limonene $0.75 \mathrm{~mL}$, methanol $1.95 \mathrm{~mL}$, and chloroform $0.3 \mathrm{~mL}$, had $g_{\text {abs }}$ values as high as +0.04 for $(R)$-limonene and -0.03 for $(S)$-limonene at the first Cotton band $(\approx 407 \mathrm{~nm})$ and weak $g_{\text {lum }}$ values of +0.004 for $(R)$-limonene and -0.003 for $(S)$-limonene at $\approx 409 \mathrm{~nm}$. The $g_{\text {abs }}$ factor, however, greatly depended on the volume fraction 
and chirality of limonene in the tersolvents. These behaviors were interpreted to the tempo-spatial stability of aggregate size induced by limonene chirality with the help of DLS measurement. Further comparative studies between $(S)$ - and $(R)$-limonene in liquid phases would be needed to verify whether our results and speculations.

\section{Acknowledgements}

M.F. learned a lot from Prof. Yoshihisa Inoue (Emeritus Prof., Osaka University) who found entropy driven chirogenesis and demonstrated circularly polarized light origin photochirogenesis along with Soai reaction. M.F. acknowledges financial supports from a Grant-in-Aid for Scientific Research (26620155, FY2014-2016), JST-CREST (FY1998-2003), and NTT (FY1990-1999). N.A.A.R. acknowledges financial support from the NAIST Presidential Special Fund. M.F. expresses a special thank his former colleagues at NTT and core members of JST-CREST, who are Prof. Hideki Sakurai (Emeritus Prof., Tohoku University), Prof. Nobuo Matsumoto (Shonan Institute of Technology), Prof. Kyozaburo Takeda (Waseda University), Prof. Hiroyuki Teramae (Josai University), Prof. Masaie Fujino (Gunma National College of Technology), Prof. Julian R. Koe (International Christian University), Dr. Kazuaki Furukawa, Dr. Seiji Toyoda, Dr. Hiroshi Nakashima, Hiromi Tamoto-Takigawa, the late Prof. Akio Teramoto (Emeritus Prof., Osaka University), Prof. Takahiro Sato (Osaka University), Prof. Ken Terao (Osaka University), Prof. Junji Watanabe (Emeritus Prof., Tokyo Institute of Technology), Prof. Kento Okoshi (Chitose Institute of Science and Technology), and Masao Motonaga (JST-CREST) for their stimulating discussion. K.Y. thanks Prof. Hiroko Yamada (NAIST), Dr. Ayako Nakao, and Takashi Matsuda for fruitful discussion and Yoshiko Nishikawa for helping him DLS measurement. Finally, we are thankful to Leigh McDowell (NAIST) for English proofreading our manuscript.

\section{Experimental}

\subsection{Materials}

Five diarylpolysilanes, $\boldsymbol{n B u P S}$, EtPS, $\boldsymbol{i P r P S}, \boldsymbol{i B u P S}$, and $\boldsymbol{t}$ BuPS, were prepared by Wurtz coupling reaction with the corresponding dichlorodiarylsilane with sodium in hot toluene in the presence of diglyme as cocatalyst. Monomers and polymers used in this work were prepared when M.F. worked for NTT-BRL and CREST-JST during1990-1999 as a part of the helical polysilane projects. A general synthetic procedure of diarylpolysilanes is given in Fig. 8 and details are given in Appendix. 


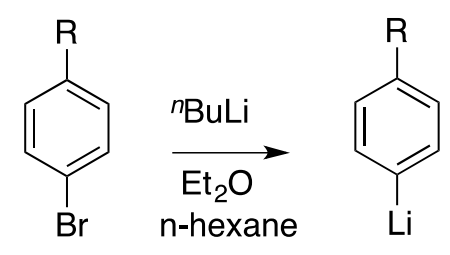

2

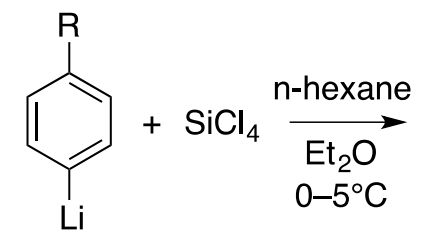<smiles>[R]c1ccc([Si](Cl)(Cl)c2ccc([R])cc2)cc1</smiles>

$2 \mathrm{Na}$ $\underset{\text { toluene }}{\stackrel{80-100^{\circ} \mathrm{C}}{\longrightarrow}}$ diglyme<smiles>[R]c1ccc([Si](C)([Si])c2ccc([R])cc2)cc1</smiles>

Fig. 8. A general synthetic scheme of diarylpolysilanes.

\subsection{Characterization methods}

Spectroscopic grade methanol and chloroform were purchased from Dojindo (Kumamoto, Japan). $(R)$ - and $(S)$-limonene, $(1 R)$ - and (1S)- $\alpha \square$ pinene, that were purchased from Tokyo Chemical Industry (TCI, Tokyo, Japan), were distilled in a reduced pressure and stored in the dark. (R)-Limonene $\left([\alpha]^{23}{ }_{\mathrm{D}}=+97.5^{\circ}\right.$ (neat)), (S)-limonene $\left([\alpha]^{23} \mathrm{D}=-98.3^{\circ}\right.$ (neat)), $(1 R)$ - $\alpha$-pinene $\left([\alpha]^{23} \mathrm{D}=+38.6^{\circ}\right.$ (neat)), and (1S)- $\alpha$-pinene $\left([\alpha]^{23}{ }_{\mathrm{D}}=-38.0^{\circ}\right.$ (neat)). The $[\alpha]^{23} \mathrm{D}$ value was obtained with a JASCO P-1020 polarimeter.

The CD and UV-vis spectra of a homogeneous solution and an aggregation suspension were simultaneously recorded on a JASCO J-725 spectropolarimeter (Hachioji-Tokyo, Japan) equipped with a Peltier controlled housing and synthetic quartz (SQ) cuvettes (GL science, Tokyo, Japan) under the condition (light source: Osram water-cooled 450-W Xenon lamp (Munich, Germany), path length: $1.0 \mathrm{~cm}$, scanning rate: $200 \mathrm{~nm} \mathrm{~min}{ }^{-1}$, bandwidth: $1 \mathrm{~nm}$, response time: $2 \mathrm{sec}$, a single accumulation, data sampling: $0.5 \mathrm{~nm}$ interval) at $25{ }^{\circ} \mathrm{C}$. UV-vis spectra were corrected by subtracting an increment in the background signals due to particle scattering by adjusting to absorbance zero at $500 \mathrm{~nm}$ in the original UV-vis data, while CD spectral data were not susceptible to particle scattering and were used without data processing. The CPL and PL spectra were recorded on a JASCO CPL-200 spectrofluoropolarimeter (Osram 150-W air-cooled Xenon lamp (Munich, Germany)), detected at an angle of $0^{\circ}$ with notch filter $\left(1: 10^{6}\right)$, a path length: $1.0 \mathrm{~cm}$ at $\approx 25{ }^{\circ} \mathrm{C}$, a scanning rate: $200 \mathrm{~nm} \min ^{-1}$, a bandwidth for excitation and monitor: $10 \mathrm{~nm}$ at $360 \mathrm{~nm}$, a band width for monitor: $10 \mathrm{~nm}$, a response time: $2 \mathrm{sec}$, no collimator lens, data sampling: 0.5 $\mathrm{nm}$ interval. Here, incident light in the CPL spectrometer used natural light that is a simultaneous irradiation of a mixture of left- and right-circularly polarized light. With a reference of quinine sulfate (in conc. $\mathrm{H}_{2} \mathrm{SO}_{4}$ ) as a standard, the quantum yield of $\boldsymbol{n B u P S}$ in tetrahydrofuran was $\approx 41 \%$. PL spectra were recorded on a JASCO FP-6500 
spectrofluorometer (a path length: $10 \mathrm{~mm}$, a band width for excitation: $3 \mathrm{~nm}$, a bandwidth for monitor: $3 \mathrm{~nm}$, a response time: $2 \mathrm{sec}$, excitation: $380 \mathrm{~nm}$, data sampling; $1.0 \mathrm{~nm}$ interval). The $n_{\mathrm{c}}(656 \mathrm{~nm})$ values of solvents, that require parameters of DLS measurements, were measured by an Atago DR-M2 thermo-controlled refractometer at $656 \mathrm{~nm}$ (Tokyo, Japan). Weight-average molecular mass $\left(M_{\mathrm{w}}\right)$ and number-average molecular mass $\left(M_{\mathrm{n}}\right)$ were obtained with Shimadzu 10A gel-permeation chromatography (GPC) (Kyoto, Japan) using an Agilent Technology Plgel mini-mix D (5 $\mu$ m, i.d. $4.6 \mathrm{~mm}$, length $20 \mathrm{~cm}$ ) or Showa Denko Shodex KF806M (10 $\mu$ m, i.d. $8.0 \mathrm{~mm}$, length $30 \mathrm{~cm})$ with special-grade tetrahydrofuran as an eluent with a calibration of polystyrene standards. Aggregate size was analyzed by Otsuka Electronics DLS-6000 (Hirakata-Osaka, Japan) detected at $633 \mathrm{~nm}$ (He-Ne laser) with an accumulation of 30 times using solution viscosity data at $25{ }^{\circ} \mathrm{C}$ obtained with a Sekonic viscometer VM-100 (Tokyo, Japan). Acquired data by these methods were re-analyzed by KaleidaGraph (ver.4.13, Synergy software, Pennsylvania, USA).

FOM images as jpeg data were obtained with a Nikon (Tokyo, Japan) Eclipse E400 with a filter block UV1A (365 nm for excitation, dichroic mirror at $400 \mathrm{~nm}$, a sharp edge, long pass filter at $400 \mathrm{~nm}$ ) under an Ushio high-pressure $\mathrm{Hg}$ lamp source (Kyoto, Japan) and a tungsten halogen lamp as white light source. ${ }^{13} \mathrm{C}(75.43 \mathrm{MHz})$ and ${ }^{29} \mathrm{Si}(59.59 \mathrm{MHz}) \mathrm{NMR}$ spectra were taken in $\mathrm{CDCl}_{3}$ at $30{ }^{\circ} \mathrm{C}$ or $40{ }^{\circ} \mathrm{C}$ with a Varian Unity $300 \mathrm{MHz}$ NMR spectrometer using tetramethylsilane as an internal standard.

\subsection{Calculation}

Molecular mechanics calculations were carried out using Molecular Simulation Inc. (MSI), the Discover 3 module, Ver. 4.00 on a Silicon Graphics Indigo II XZ computer. Standard parameters (default) were $\mathrm{Si}-\mathrm{Si}$ bond length of $2.34 \AA$, an $\mathrm{Si}-\mathrm{Si}-\mathrm{Si}$ bond angle of $111^{\circ}$, and the pcff force field (MSI) suited for polymers. MSI built-in functions were used as the set-up parameters, including simple-minimization under default conditions with dihedral angle restraints, steepest descents for the first derivative, iteration limit of 1000 , movement limit of 0.2 , and derivative for 1.0 and simple dynamics with a constant volume and temperature, initial temperature of $300 \mathrm{~K}$, direct velocity scaling with a time step of $1 \mathrm{fs}$, integration, and initial velocity of random velocities from the Boltzmann distribution.

\section{References}

1. L. Roth, J. Saur, K. D. Retherford, D. F. Strobel, P. D. Feldman, M. A. McGrath, F. Nimmo, Science, 343 (2013) 171-174. 
2. F. Pepe et al. Astron. Astrophys. 534 (2011) A58 (16 pages).

3. E. V. Quintana et al., Science, 344 (2014) 277-280.

4. M. Tuomi et al., Astron. Astrophys. 551 (2013) A29 (21 pages).

5. L. Iess et al., Science, 344 (2014) 78-80.

6. R. D. Lorenz et al., Icarus, 237 (2014) 9-15.

7. F. Goesmann et al., Science, 349 (2015) aab0689.

8. J.-P. Birbring et al., Science, 349 (2015) aab0671.

9. L. Ojha, M. B. Wilhelm, S. L. Murchie, A. S. McEwen, J. J. Wray, J. Hanley, M Massé, M. Chojnacki, Nat. Geosci., 8 (2015) 829-832.

10. A. J. Macdermott, Chirality, 24 (2012) 764-769.

11. W.B. Sparksa, et al., J. Quant. Spectrosc. Radiat. Transfer, 110 (2009) 1771-1779.

12. R. Plasson, D. K. Kondepudi, H. Bersini, A. Commeyras , K. Asakura, Chirality, 19 (2007) 589-600.

13. E. Schrodinger, What is life?: With mind and matter and autobiographical sketches (Cambridge Univ. Press) 1944.

14. V. A. Avetisov, V. I. Goldanskii, V. V. Kuz'min. Phys. Today, 44 (1991) 33-41.

15. W. A. Bonner, Orig. Life Evol. Biosph., 21 (1992) 407-420.

16. Y. Inoue, Chem. Rev., 92 (1992) 741-770.

17. B. L. Feringa, R. A. van Delden, Angew. Chem. Int. Ed., 38 (1999) 3418-3438.

18. J. Bailey, A. Chrysostomou A, J. H. Hough, T. M. Gledhill, A. McCall, S. Clark, F. Ménard F., M. Tamura, Science, 281 (1998) 672-674.

19. H. Nishino, A. Kosaka, G. A. Hembury, F. Aoki, K. Miyauchi, H. Shitomi, H. Onuki, Y. Inoue, J. Am. Chem. Soc., 124 (2002) 11618-11627.

20. T. Kawasaki, M. Sato, S. Ishiguro, T. Saito, Y. Morishita, I. Sato, H. Nishino, Y. Inoue, K. Soai J. Am. Chem. Soc., 127 (2005) 3274-3275.

21. C. Meinert, S. V. Hoffmann, P. Cassam-Chenaï, A. C. Evans, C. Giri, L. Nahon, U. J. Meierhenrich, Angew. Chem. Int. Ed. 53 (2014) 210-214.

22. D. G. Blackmond, Proc. Nat. Sci. Acad. USA, 101 (2004) 5732-5736.

23. M. Fujiki, Chem. Rec., 9 (2009) 271-298.

24. M. Liu, L. Zhang, T. Wang, Chem. Rev., 115 (2015) 7304-7397.

25. M. H. Engel, B. Nagy, Nature, 296 (1982) 837-840.

26. S. Epstein, R. V. Krishnamurthy, J. R. Cronin, S. Pizzarello, G. U. Yuen, Nature 326 (1987) 477-479. 
27. M. H. Engel, S. A. Macko, Nature 389 (1997) 265-268.

28. A. Córdova, M. Engqvist, I. Ibrahem, J. Casa, H. Sundén, Chem. Commun., (2005) 2047-2049.

29. I. Sato, H. Urabe, S. Ishiguro, T. Shibata, K. Soai, Angew. Chem. Int. Ed., 42 (2003) 315317.

30. Y. Ueno, K. Yamada, N. Yoshida, S. Maruyama, Y. Isozaki, Nature, 440 (2006) 516-519.

31. S. J. Nojzsis, G. Arrhenius, K. D. McKeegan, T. M. Harrison, A. P. Nutman, C. R. Friend, Nature, 384 (1996) 55-59.

32. Y. Okamoto, T. Kakegawa, A. Ishida, T. Nagase, M. T. Rosing, Nat. Geosci., 7 (2014) 25-28.

33. J. L. Kirschvink, E. J. Gaidos, L. E. Bertani, N. J. Beukes, J. Gutzmer, L. N. Maepa, R. E. Steinberger, Proc. Nat. Sci. Acad. USA, 97 (2000) 1400-1405.

34. A. D. Rooney, F. A. Macdonald, J. V. Strauss, F. Ö. Dudás, C. Hallmann, D. Selby, Proc. Nat. Sci. Acad. USA, 111 (2014) 51-56.

35. P. F. Hoffman, D. P. Schrag, Terra Nova, 14 (2002) 129-155.

36. A. I. Oparin. Life: Its nature, origin, and development, Academic Press (New York, 1961).

37. J. B. S. Haldane, New Biol., 16 (1954) 12-27.

38. H. Staudinger, Ber. Deut. Chem. Ges., 53 (1920) 1073-1085.

39. H. Nakashima, M. Fujiki, J. R. Koe, M. Motonaga, J. Am. Chem. Soc., 123 (2001) 1963-1969.

40. M. Fujiki, M. Macromol. Rapid Commun., 22 (2001) 539-563.

41. H. Goto, E. Yashima, J. Am. Chem. Soc., 124 (2002) 7943-7949.

42. B. M. W. Langeveld-Voss, R. J. M. Waterval, R. A. J. Janssen, E. W. Meijer, Macromolecules, 32 (1999) 227-230.

43. X. Liu, J. Jiao, X. Jiang, J. Li, Y. Cheng, C. Zhu, J. Mater. Chem. C., 1 (2013) 4713-4719.

44. Y. Kawagoe, M. Fujiki, Y. Nakano, New J. Chem., 34 (2010) 637-647.

45. Y. Nakano, M. Fujiki, Macromolecules, 44 (2011) 7511-7519.

46. J. Liu, H. Su, L. Meng, Y. Zhao, C. Deng, J. C. Y. Ng, P. Lu, M. Faisal, J. W. Y. Lam, X. Huang, H. Wu, K. S. Wong, B. Z. Tang, Chem. Sci., 3 (2012) 2737-2747.

47. N. Suzuki, M. Fujiki, R. Kimpinde-Kalunga, J. R. Koe, J. Am. Chem. Soc., 135 (2013) 13073-13079. 
48. M. Fujiki, Y. Donguri, Y. Zhao, A. Nakao, N. Suzuki, K. Yoshida, W. Zhang, Polym. Chem., 6 (2015) 1627-1638.

49. Y. Nakano, Y. Liu, M. Fujiki, Polym. Chem., 1 (2010) 460-469.

50. Y. Nakano, F. Ichiyanagi, M. Naito, Y. G. Yang, M. Fujiki, Chem. Commun., 48 (2012) 6636-6638.

51. M. Fujiki, A. J. Jalilah, N. Suzuki, M. Taguchi, W. Zhang, M. M. Abdellatif, K. Nomura, RSC Adv., 2 (2012) 6663-6671.

52. M. Fujiki, Y. Kawagoe, Y. Nakano, A. Nakao, Molecules, 18 (2013) 7035-7057.

53. L. Wang, N. Suzuki, J. Liu, T. Matsuda, N. A. A. Rahim, W. Zhang, M. Fujiki, Z. Zhang, N. Zhou, X. Zhu, Polym. Chem., 5 (2014) 5920-5927.

54. J. Liu, J. Zhang, S. Zhang, N. Suzuki, M. Fujiki, L. Wang, L. Li, W. Zhang, N. Zhou, X. Zhu, Polym. Chem., 5 (2014) 784-791.

55. R. D. Miller, R. Sooriyakumaran, J. Polym. Sci. Polym. Lett. Ed., 25 (1987) 321-326.

56. P. M. Cotts, R. D. Miller, R. Sooriyakumaran, Silicon-Based Polymer Science, Chapter 23, 1989, 397-412, J. M. Zeigler, F. W. G. Fearon (eds), Adv. Chem., Vol. 224.

57. J. R. Koe, M. Fujiki, H. Nakashima, M. Motonaga, Synthetic Macromolecules with Higher Structural Order, Chapter 6, 2002, 67-86, ACS Symposium Series, Vol. 812.

58. M. Fujiki, J. R. Koe, K. Terao, T. Sato, A. Teramoto, J. Watanabe, Polym. J., 35 (2003) 297-344.

59. K. Takeda, H. Teramae, N. Matsumoto, J. Am. Chem. Soc., 108 (1986) 8186-8190.

60. M. Fujiki, J. Am. Chem. Soc., 116 (1994) 6017-6018.

61. S. Toyoda, M. Fujiki, H. Suzuki, N. Matsumoto, Solid State Commun., 103 (1997) 87-89.

62. F. P. Ureña, J. R. A. Moreno, J. J. L. González Tetrahedron: Asymmetry, 20 (2009) 89-97.

63. F. Reinscheid, U.M. Reinscheid, J. Mol. Struct., 1106 (2016) 141-153.

64. D. W. Deamer, R. Dick, W. Thiemann, M. Shinitzky, Chirality, 19 (2007) 751-763.

65. M. Shinitzky, Chirality, 25 (2013) 308-311.

66. E. K. Kodona, C. Alexopoulos, E. Panou-Pomonis, P. J. Pomonis, J. Colloid Interface Sci., 319 (2008) 71-80.

67. M. Fujiki, Symmetry, 2 (2010) 1625-1652.

68. R. A. Hegstrom, D. K. Kondepudi, Sci. Am., 262 (1990) 98-105.

69. R. A. Hegstrom, D. W. Rein, P. G. H. Sandars, J. Chem. Phys., 73 (1980) 2329-2341.

70. R. N. Compton, R. M. Pagni, Adv. At. Mol. Opt. Phys., 48 (2002) 219-261. 
71. V. I. Goldanskii, V. V. Kuz'min, Sov. Phys. Usp., 32 (1989) 1-29.

72. D. B. Cline, Symmetry, 2 (2010) 1450-1460.

73. W. A. Bonner, Top. Stereochem. 18 (1991) 1-96.

74. E. Kearns, T. Kajita, Y. Totsuka, Sci. Am., 281 (1999) 64-71.

75. A. B. McDonald, J. R. Klein, D. L. Wark, Sci. Am., 16 (2005) 22-31.

76. B. Aubert et al., Phys. Rev. Lett., 87 (2001) 091801 (8 pages).

77. K. Abe et al., Phys. Rev. Lett., 87 (2001) 091802 (7 pages).

78. L. D. Barron, Molecular Light Scattering and Optical Activity (2nd ed) (Cambridge Univ. Press, 2009).

79. M. Quack, Angew. Chem. Int. Ed. Engl., 28 (1989) 571-586.

80. J. K. Laerdahl, P. Schwerdtfeger, Phys. Rev. A, 60 (1999) 4439-4453. 
$\mathrm{CH}_{3} \widehat{\bigcap}$ evolution of helix propagation of chiral aggregates

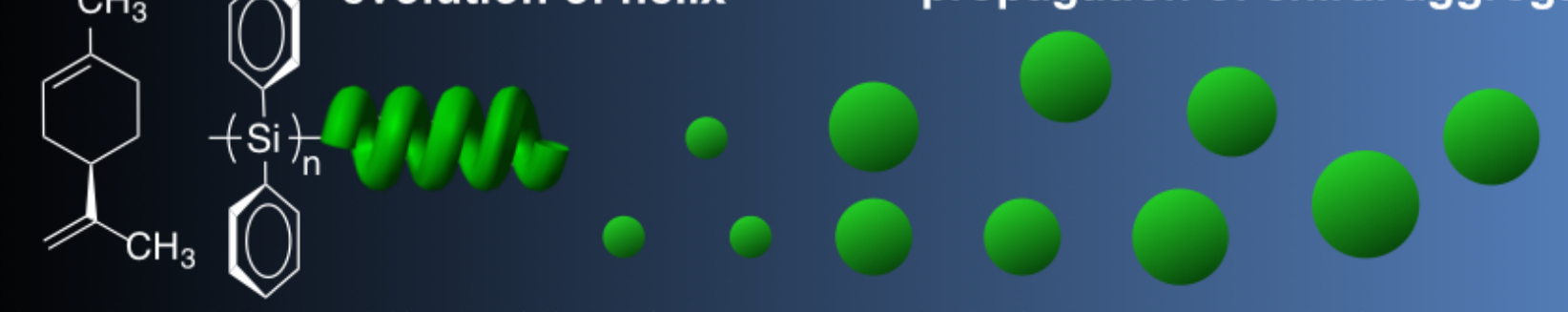

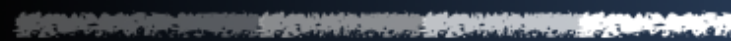

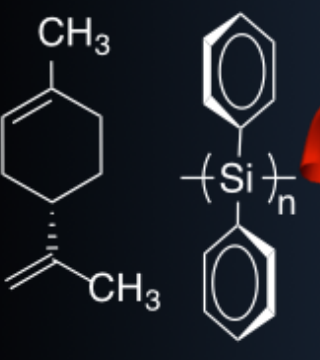

